\title{
Rh-Catalyzed Enantioselective Diboration of Simple Alkenes: Reaction Development and Substrate Scope
}

\author{
Stéphane Trudeau, Jeremy B. Morgan, Mohanish Shrestha and James P. Morken* \\ Department of Chemistry, Venable and Kenan Laboratories, The University of North \\ Carolina at Chapel Hill, Chapel Hill, North Carolina 27599-3290 \\ Supplementary Material
}

\section{Table of contents}

1) General remarks .............................S 1

2) Experimental details ...................... S 2

3) ${ }^{1} \mathrm{H}$ and ${ }^{13} \mathrm{C}$ NMR spectra ............... S 22 


\section{1) General remarks.}

${ }^{1} \mathrm{H}$ NMR chemical shifts are reported in ppm from tetramethylsilane with the solvent resonance as the internal standard $\left(\mathrm{CDCl}_{3}: 7.24 \mathrm{ppm}\right)$. Data are reported as follows: chemical shift, integration, multiplicity $(\mathrm{s}=$ singlet, $\mathrm{d}=$ doublet, $\mathrm{t}=$ triplet, $\mathrm{q}=$ quartet, $\mathrm{br}=$ broad, $\mathrm{m}=$ multiplet), coupling constants $(\mathrm{Hz})$ and assignment. ${ }^{13} \mathrm{C} \mathrm{NMR}$ chemical shifts are reported in ppm from tetramethylsilane with the solvent as the internal standard $\left(\mathrm{CDCl}_{3}: 77.0 \mathrm{ppm}\right)$.

Liquid chromatography was performed using forced flow (flash chromatography) on silica gel $\left(\mathrm{SiO}_{2}, 32\right.$ to $\left.63 \mu \mathrm{m}\right)$. Thin layer chromatography (TLC) was performed on $0.25 \mathrm{~mm}$ silica gel plates.

Analytical gas-liquid chromatography (GLC) was performed on a Supelco $\beta$-dex 120 column with helium as the carrier gas. Analytical high performance liquid chromatography (HPLC) was performed using a Daicel Chiralcel OD-H column. Analytical supercritical fluid chromatography (SFC) was performed using a Daicel Chiralcel OD-H column.

All reactions were conducted in oven and flame dried glassware under an inert atmosphere of argon. Alkene starting materials were all commercially available unless otherwise described. All reagents were used as received. 


\section{2) Experimental details.}

\section{Representative Procedure for Catalytic, Enantioselective Diboration Reaction.}

An oven-dried $20 \mathrm{~mL}$ vial equipped with a stir-bar was charged with $6.1 \mathrm{mg}$ $(0.021 \mathrm{mmol})$ of (bicyclo[2.2.1]hepta-2,5-diene)-(2,4-pentanedionato)-rhodium (I) ((nbd)Rh(acac)), $9.2 \mathrm{mg}(0.021 \mathrm{mmol})$ of (S)-Quinap, and $1.7 \mathrm{~mL}$ of THF under an inert atmosphere of argon in a dry-box. The resultant yellow solution was stirred for 5 minutes. After this time, $148 \mathrm{mg}(0.62 \mathrm{mmol})$ of bis(catecholato)diboron was added to the solution under argon. The solution turned immediately from yellow to dark brownish-red. The solution was allowed to stir for 5 minutes. After this time, $84 \mathrm{mg}(0.42 \mathrm{mmol})$ of trans-(7methyl-octa-1,6-dienyl)-benzene was added to the solution under argon. The vial was sealed with a screw-cap and removed from the dry box, where the solution was allowed to stir for 14 hours at ambient temperature. After this time, the mixture was cooled to $0^{\circ} \mathrm{C}$ and $1.25 \mathrm{~mL}$ of $3 \mathrm{M} \mathrm{NaOH}$ and then $0.800 \mathrm{~mL}$ of $30 \% \mathrm{H}_{2} \mathrm{O}_{2}$ (dropwise with caution) were added under nitrogen. The solution was allowed to stir at ambient temperature for 6 hours. The solution was then quenched with $2 \mathrm{~mL}$ of saturated aqueous $\mathrm{Na}_{2} \mathrm{~S}_{2} \mathrm{O}_{3}$ and 10 $\mathrm{mL}$ of $1 \mathrm{M} \mathrm{NaOH}$. The mixture was extracted with ethyl acetate $(3 \times 25 \mathrm{~mL})$ and the combined organic layers were washed with brine $(1 \times 10 \mathrm{~mL})$. The organic layers were then dried over anhydrous $\mathrm{MgSO}_{4}$, filtered, and the solvent removed by rotary evaporation. The crude material was purified by silica gel chromatography $(9: 1$ to $6: 4$ hexanes/ethyl acetate) to provide $63 \mathrm{mg}(64 \%)$ of pure $(1 R, 2 R)$-7-methyl-1-phenyl-oct6-ene-1,2-diol. 
Compounds from table 4 (entries 1-3, 8-10), table 5 (entry 3) and table 6 (entry 4 ) were all previously reported ${ }^{1}$. Compounds from table 5 (entries 1-2, 4-7) were also previously reported ${ }^{2}$.

\section{Table 4, entry 4}<smiles>CCCCOCCC(O)C(O)C[Mg]</smiles>

(3R, 4R)-1-(tert-butyldiphenylsilanyloxy)-hexane-3,4-diol ${ }^{3}$. IR (neat, $\left.v \mathrm{~cm}^{-1}\right)$ : 3481, 2964, 2933, 1640, 1472, 1428, 1391; ${ }^{1} \mathrm{H}$ NMR: $\delta$ 7.68-7.66 (4H, m), 7.45-7.37 (6H, m), $3.88(2 \mathrm{H}, \mathrm{t}, J=5.1 \mathrm{~Hz}), 3.77-3.73(1 \mathrm{H}, \mathrm{m}), 3.41(1 \mathrm{H}, \mathrm{d}, J=3.4 \mathrm{~Hz}), 3.39-3.33(1 \mathrm{H}, \mathrm{m})$, $2.58(1 \mathrm{H}, \mathrm{d}, J=5.4 \mathrm{~Hz}), 1.84-1.77(1 \mathrm{H}, \mathrm{m}), 1.71-1.64(1 \mathrm{H}, \mathrm{m}), 1.59-1.44(2 \mathrm{H}, \mathrm{m}), 1.05$ (9H, s), $0.98(3 \mathrm{H}, \mathrm{t}, J=7.4 \mathrm{~Hz}) ;{ }^{13} \mathrm{C}$ NMR: $\delta 135.5,132.8,129.9,127.8,75.8,73.5$, $62.8,35.1,26.8,26.3,19.0,10.1$.

Proof of Stereochemistry. Stereochemical ratios were determined in comparison to authentic racemic materials prepared by osmium tetraoxide catalyzed dihydroxylation. Absolute stereochemistry established in comparison to authentic $(3 S, 4 S)$ isomer prepared via a Sharpless asymmetric dihydroxylation (Becker, H.; King, S. B.; Taniguchi, M.; Vanhessche, K.; Sharpless, K. B. J. Org. Chem. 1995, 60, 3940). 
SFC (OD-H, $150 \mathrm{psi}, 40^{\circ} \mathrm{C}$, flow $\left.=2 \mathrm{~mL} / \mathrm{min}, 1.5 \% \mathrm{MeOH}\right)$ analysis of the product:

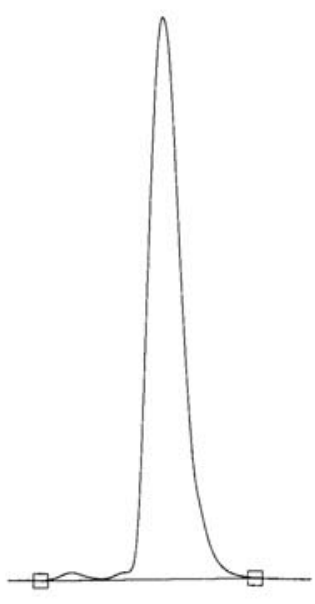

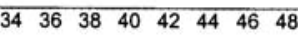

Diboration

product

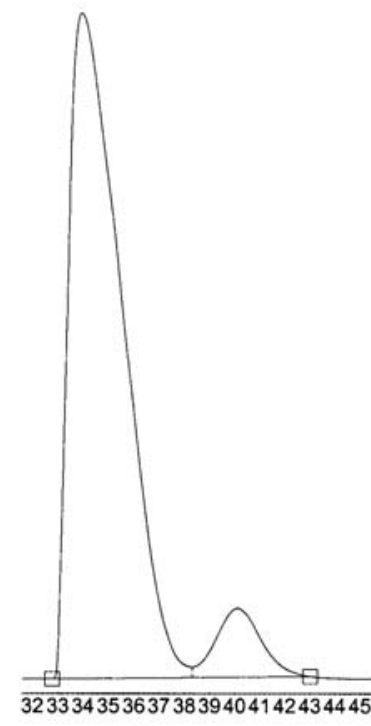

Authentic

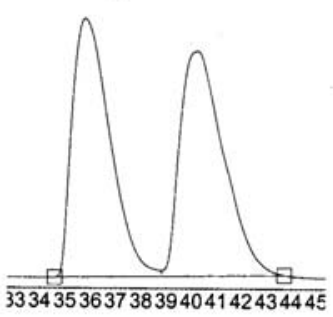

Racemic

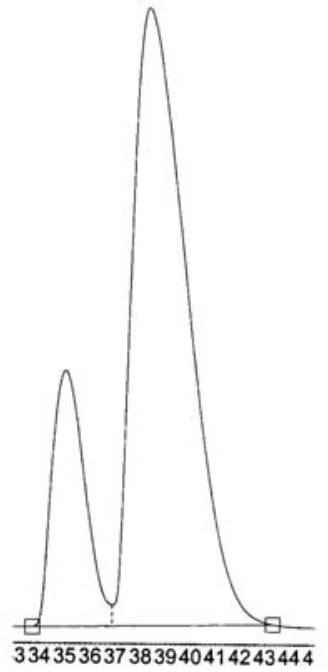

Racemic +

product

Table 4, entry 5<smiles>CCCCOCC[C@H](O)[C@@H](O)CC</smiles>

(3R, 4R)-1-(tert-butyldimethylsilanyloxy)-hexane-3,4-diol ${ }^{4}$. IR (neat, $v \mathrm{~cm}^{-1}$ ): 3481, 2958, 2933, 1640, 1472, 1389; ${ }^{1} \mathrm{H}$ NMR: $\delta$ 3.90-3.80 (2H, m), 3.70-3.65 (1H, m), 3.50 $(1 \mathrm{H}, \mathrm{d}, J=3.4 \mathrm{~Hz}), 3.36-3.31(1 \mathrm{H}, \mathrm{m}), 2.54(1 \mathrm{H}, \mathrm{d}, J=5.5 \mathrm{~Hz}), 1.81-1.72(1 \mathrm{H}, \mathrm{m}), 1.69-$ $1.62(1 \mathrm{H}, \mathrm{m}), 1.57-1.42(2 \mathrm{H}, \mathrm{m}), 0.96(3 \mathrm{H}, \mathrm{t}, J=7.5 \mathrm{~Hz}), 0.87(9 \mathrm{H}, \mathrm{s}), 0.06(6 \mathrm{H}, \mathrm{s}) ;{ }^{13} \mathrm{C}$ NMR: $\delta 75.7,73.8,62.0,35.2,26.3,25.8,18.1,10.1,-5.6$. 
Proof of Stereochemistry. Stereochemical ratios were determined in comparison to authentic racemic materials prepared by osmium tetraoxide catalyzed dihydroxylation.

Chiral GLC ( $\beta$-dex, Supelco, $100^{\circ} \mathrm{C}, 20$ psi) analysis of the product:

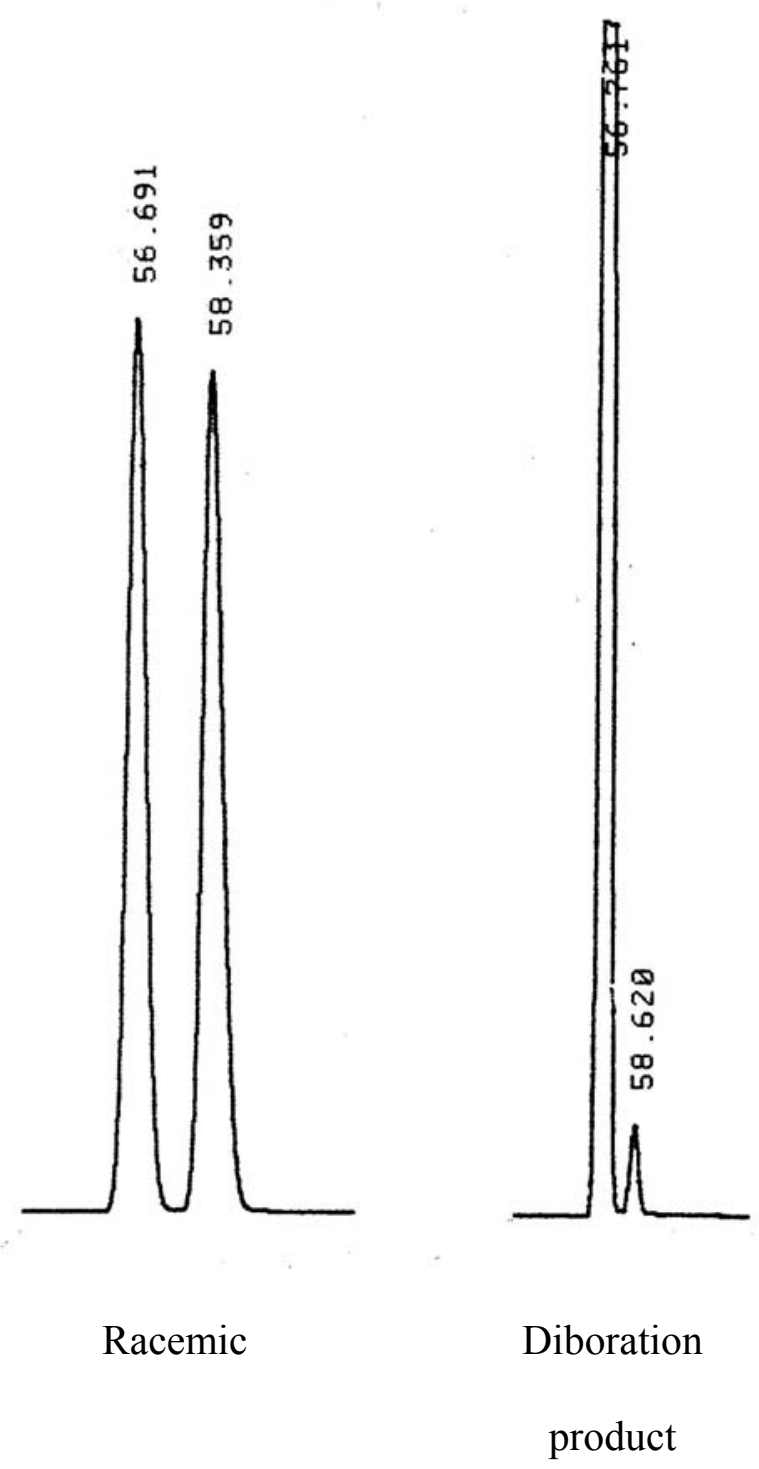




\section{Table 4, entry 6}<smiles>CC[C@H](O)[C@@H](O)CCOC</smiles>

(3R, 4R)-1-methoxymethoxy-hexane-3,4-diol ${ }^{5}$. IR (neat, $v \mathrm{~cm}^{-1}$ ): 3483, 3056, 2989, 2968, 2939, 1640, 1465, 1422; ${ }^{1} \mathrm{H}$ NMR: $\delta 4.62(2 \mathrm{H}, \mathrm{s}), 3.78-3.71(2 \mathrm{H}, \mathrm{m}), 3.67-3.63$ $(1 \mathrm{H}, \mathrm{m}), 3.39-3.33(1 \mathrm{H}, \mathrm{m}), 3.36(3 \mathrm{H}, \mathrm{s}), 2.85(1 \mathrm{H}, \mathrm{d}, J=4.0 \mathrm{~Hz}), 2.30(1 \mathrm{H}, \mathrm{d}, J=5.3$ $\mathrm{Hz}), 1.83-1.77(2 \mathrm{H}, \mathrm{m}), 1.60-1.42(2 \mathrm{H}, \mathrm{m}), 0.98(3 \mathrm{H}, \mathrm{t}, J=7.5 \mathrm{~Hz}) ;{ }^{13} \mathrm{C}$ NMR: $\delta 96.6$, $75.8,72.8,65.8,55.4,33.2,26.4,10.1$.

Proof of Stereochemistry. Stereochemical ratios were determined in comparison to authentic racemic materials prepared by osmium tetraoxide catalyzed dihydroxylation. 
Chiral GLC ( $\beta$-dex, Supelco, $\left.100^{\circ} \mathrm{C}, 20 \mathrm{psi}\right)$ analysis of the product:

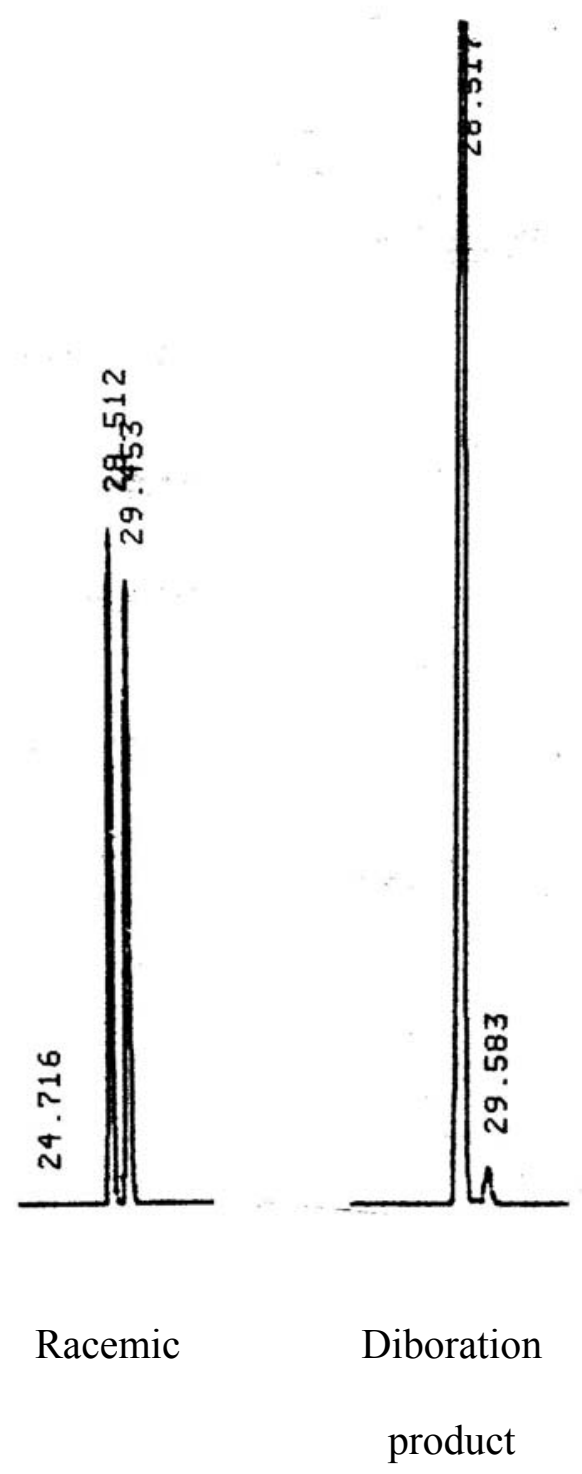

Table 4, entry 7<smiles>CCC(O)C(O)CCOCc1ccccc1</smiles>

(3R, 4R)-1-benzyloxy-hexane-3,4-diol ${ }^{6}$. IR (neat, $v \mathrm{~cm}^{-1}$ ): 3481, 3056, 2968, 2937, 2877, 1640, 1455, 1422, 1364; ${ }^{1} \mathrm{H}$ NMR: $\delta$ 7.36-7.28 (5H, m), $4.51(2 \mathrm{H}, \mathrm{s}), 3.74-3.65$ 
$(3 \mathrm{H}, \mathrm{m}), 3.36-3.31(1 \mathrm{H}, \mathrm{m}), 3.09(1 \mathrm{H}, \mathrm{d}, J=3.7 \mathrm{~Hz}), 2.41(1 \mathrm{H}, \mathrm{d}, J=5.3 \mathrm{~Hz}), 1.91-1.82$

$(1 \mathrm{H}, \mathrm{m}), 1.79-1.72(1 \mathrm{H}, \mathrm{m}), 1.60-1.41(2 \mathrm{H}, \mathrm{m}), 0.97(3 \mathrm{H}, \mathrm{t}, J=7.5 \mathrm{~Hz}) ;{ }^{13} \mathrm{C}$ NMR: $\delta$ $137.7,128.5,127.8,127.7,75.7,73.4,73.1,68.5,33.2,26.3,10.1$.

Proof of Stereochemistry. Stereochemical ratios were determined in comparison to authentic racemic materials prepared by osmium tetraoxide catalyzed dihydroxylation.

SFC (OD-H, $150 \mathrm{psi}, 40^{\circ} \mathrm{C}$, flow $\left.=2 \mathrm{~mL} / \mathrm{min}, 2.0 \% \mathrm{MeOH}\right)$ analysis of the product:

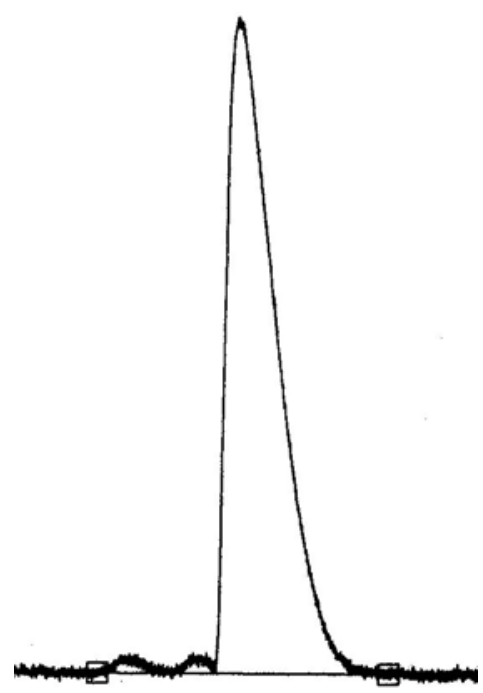

25 30

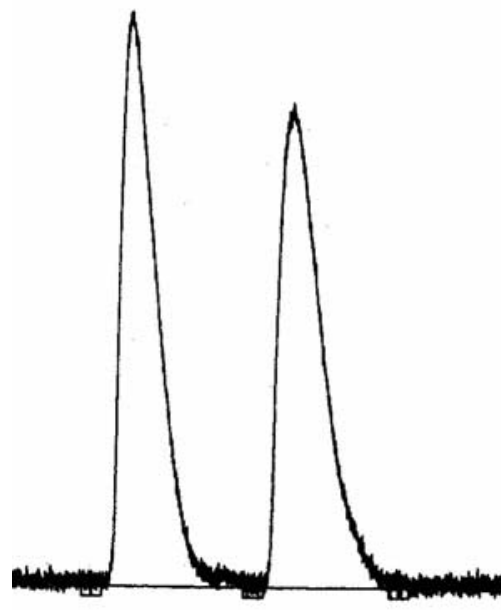

25

Racemic

product

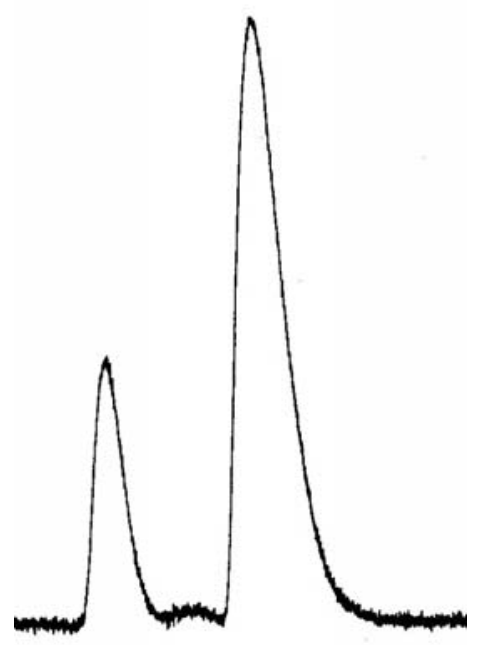

25

30

Diboration product 


\section{Table 5, entry 8}

$t$-butyl $\overbrace{}^{\mathrm{OH}} \mathrm{OH}$

(2S)-4,4-dimethylpentane-1,2-diol. IR (neat, $v \mathrm{~cm}^{-1}$ ): 3365, 2954, 2871, 1468, 1366, 1088, 1042; ${ }^{1} \mathrm{H}$ NMR: $\delta 3.79(1 \mathrm{H}, \mathrm{m}), 3.51(1 \mathrm{H}, \mathrm{dd}, J=11.1,3.2 \mathrm{~Hz}), 3.33(1 \mathrm{H}, \mathrm{dd}, J=$ 11.1, $8.4 \mathrm{~Hz}), 3.14(2 \mathrm{H}, \mathrm{s}), 1.31(1 \mathrm{H}, \mathrm{dd}, J=14.6,7.7 \mathrm{~Hz}), 1.20(1 \mathrm{H}, \mathrm{dd}, J=14.5,2.9$ $\mathrm{Hz}), 0.93(9 \mathrm{H}, \mathrm{s}) ;{ }^{13} \mathrm{C} \mathrm{NMR}: \delta$ 69.9, 68.0, 46.8, 30.0, 30.0; HRMS (CI) $\left(\mathrm{M}+\mathrm{NH}_{4}\right)^{+}$calc'd for $\mathrm{C}_{7} \mathrm{H}_{20} \mathrm{NO}_{2}: 150.1489$. Found: 150.1497 .

Proof of Stereochemistry. Stereochemical ratios were determined in comparison to authentic racemic materials prepared by osmium tetraoxide catalyzed dihydroxylation. Absolute stereochemistry established in comparison to authentic $(2 S)$ isomer prepared via a Sharpless asymmetric dihydroxylation (Becker, H.; King, S. B.; Taniguchi, M.; Vanhessche, K.; Sharpless, K. B. J. Org. Chem. 1995, 60, 3940). 
SFC (OD-H, $150 \mathrm{psi}, 50{ }^{\circ} \mathrm{C}$, flow $\left.=3 \mathrm{~mL} / \mathrm{min}, 0 \% \mathrm{MeOH}\right)$ analysis of the benzoate diester (BzCl / cat. DMAP, pyr) product:

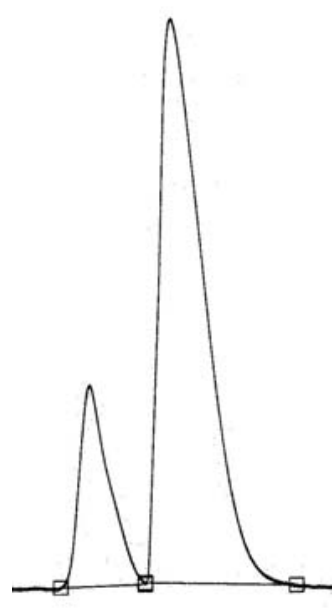

$\begin{array}{lllllllll}22 & 24 & 26 & 28 & 30 & 32 & 34 & 36 & 38\end{array}$

Diboration

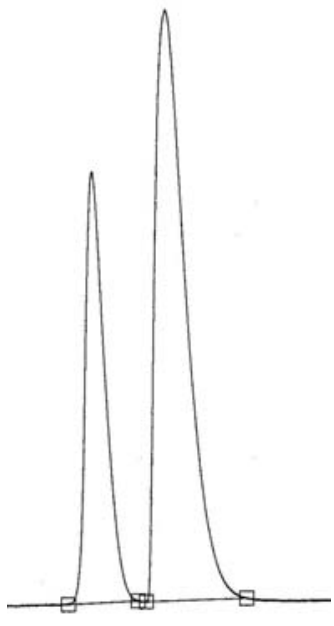

$\begin{array}{lllllllll}18 & 20 & 22 & 24 & 26 & 28 & 30 & 32 & 34\end{array}$

Authentic
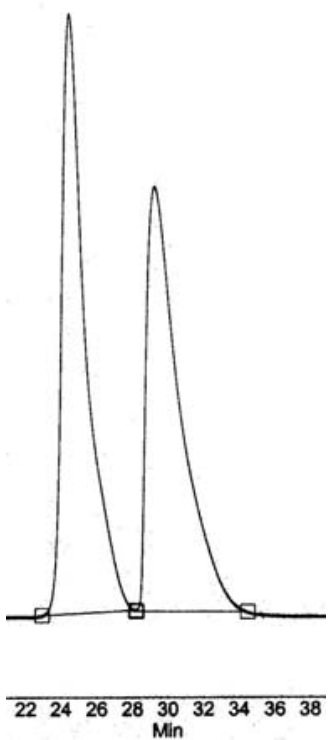

Racemic

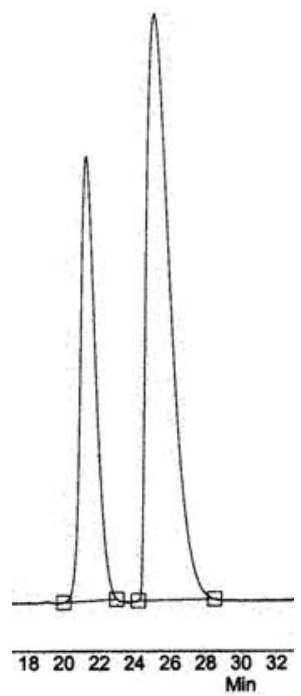

Racemic +

Diboration

product

Table 5, entry 9 (substrate)<smiles></smiles>

1-((2,2-dimethylpent-4-enyloxy)methyl)benzene. Sodium hydride (60\% in mineral oil) (67 mg, $1.68 \mathrm{mmol}$ ) was added to a round bottom flask and washed three times with pentane. THF $(3.0 \mathrm{~mL})$ was then added and the resulting suspension was cooled to $0^{\circ} \mathrm{C}$. A solution of 2,2-dimethylpent-4-en-1-ol ${ }^{7}(101 \mathrm{mg}, 0.88 \mathrm{mmol})$ in THF $(3.0 \mathrm{~mL})$ was then added dropwise and the mixture was stirred $1 \mathrm{~h} 30$ at room temperature. Benzyl bromide $(0.16 \mathrm{~mL}, 1.33 \mathrm{mmol})$ and tetrabutylammonium iodide $(33 \mathrm{mg}, 8.8 \mu \mathrm{mol})$ were then added and mixture was stirred $16 \mathrm{~h}$ at room temperature. A saturated $\mathrm{NH}_{4} \mathrm{Cl}$ aqueous 
solution $(10 \mathrm{~mL})$ was then added and mixture was extracted three times with $\mathrm{Et}_{2} \mathrm{O}$. The combined organic phases were dried with $\mathrm{MgSO}_{4}$, filtered and concentrated under reduced pressure. The crude product was purified by flash chromatography (100/0 to 95/5: hex/EtOAc) to yield the product $(0.16 \mathrm{~g}, 88 \%)$ as a clear oil. IR (neat, $\left.v \mathrm{~cm}^{-1}\right)$ : 2958, 2860, 1102; ${ }^{1} \mathrm{H}$ NMR: $\delta$ 7.40-7.26 (5H, m), 5.87-5.76 (1H, m), 5.05-5.04 (1H, m), 5.04-5.00 (1H, m), $4.52(2 \mathrm{H}, \mathrm{s}), 3.15(2 \mathrm{H}, \mathrm{s}), 2.08(2 \mathrm{H}, \mathrm{td}, J=7.5,1.1 \mathrm{~Hz}), 0.93(6 \mathrm{H}, \mathrm{s})$;

${ }^{13}$ C NMR: $\delta 139.1,135.4,128.2,127.3,127.3,116.9,79.1,73.2,43.7,34.9,24.5$.

\section{Table 5, entry 9}<smiles>[B]OCC(C)(C)CC(C)(C)C[C@H](O)CO</smiles>

(2R)-5-(benzyloxy)-4,4-dimethylpentane-1,2-diol. IR (neat, $v \mathrm{~cm}^{-1}$ ): 3394, 2954, 2929, 2871, 1096; ${ }^{1} \mathrm{H}$ NMR: $\delta$ 7.36-7.26 (5H, m), 4.52 (2H, d, $\left.J=1.4 \mathrm{~Hz}\right), 3.82-3.76(1 \mathrm{H}, \mathrm{m})$, 3.49 (1H, dd, $J=10.9,3.5 \mathrm{~Hz}), 3.41-3.36(3 \mathrm{H}, \mathrm{m}), 3.27(1 \mathrm{H}, \mathrm{d}, J=9.0 \mathrm{~Hz}), 3.23(1 \mathrm{H}, \mathrm{dd}$, $J=9.0,0.2 \mathrm{~Hz}), 1.47(1 \mathrm{H}, \mathrm{dd}, J=14.8,9.7 \mathrm{~Hz}), 1.32(1 \mathrm{H}, \mathrm{dd}, J=14.7,1.3 \mathrm{~Hz}), 0.99$ (3H, s), $0.90(3 \mathrm{H}, \mathrm{s}) ;{ }^{13} \mathrm{C}$ NMR: $\delta 137.8,128.9,128.3,128.2,80.0,74.0,68.8,68.1,45.2$, 34.5, 28.4, 24.2; $\mathrm{MS}(\mathrm{ESI})(\mathrm{M}+\mathrm{Na})^{+}$calc'd for $\mathrm{C}_{14} \mathrm{H}_{22} \mathrm{O}_{3} \mathrm{Na}$ : 261.1. Found: 261.1.

Proof of Stereochemistry. Stereochemical ratios were determined in comparison to authentic racemic materials prepared by osmium tetraoxide catalyzed dihydroxylation. Absolute stereochemistry established in comparison to authentic $(2 R)$ isomer prepared via a Sharpless asymmetric dihydroxylation (Becker, H.; King, S. B.; Taniguchi, M.; Vanhessche, K.; Sharpless, K. B. J. Org. Chem. 1995, 60, 3940). 
Chiral GLC ( $\beta$-dex, Supelco, $120^{\circ} \mathrm{C} 178 \mathrm{~min}$ then $\uparrow 1^{\circ} \mathrm{C} / \mathrm{min}$ to $180^{\circ} \mathrm{C}$, $20 \mathrm{psi}$ ) analysis of the acetonide (dimethoxypropane/cat. pTsOH) product:

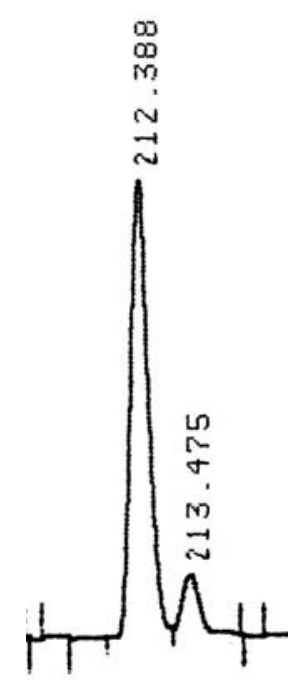

Diboration

product

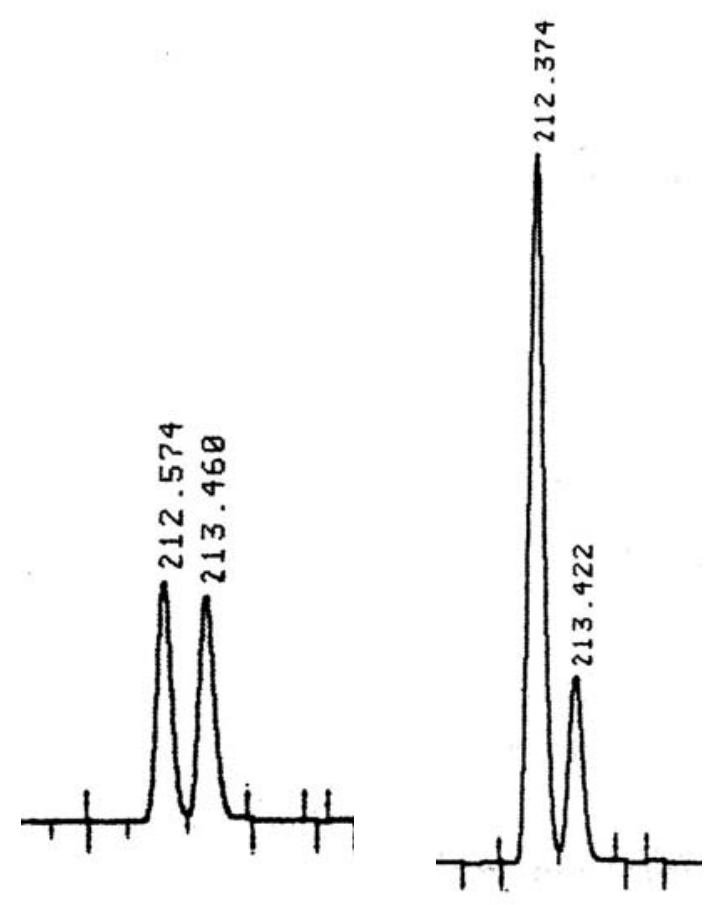

Racemic

Racemic +

Diboration

Product

Table 5, entry 10<smiles>[B-]#[R5]OCC(C)(C)CC(O)CO</smiles>

(2R)-5-(tert-butyldiphenylsilyloxy)-4,4-dimethylpentane-1,2-diol ${ }^{8}$. IR (neat, $v \mathrm{~cm}^{-1}$ ): 3381, 2958, 2931, 2894, 2858, 1111, 1090; ${ }^{1} \mathrm{H}$ NMR: $\delta$ 7.67-7.64 (4H, m), 7.45-7.36 (6H, m), 3.89-3.84 (1H, m), $3.54(1 \mathrm{H}, \mathrm{dd}, J=10.9,3.5 \mathrm{~Hz}), 3.45-3.38(3 \mathrm{H}, \mathrm{m}), 3.15(2 \mathrm{H}, \mathrm{br}$ s), $1.51(1 \mathrm{H}, \mathrm{dd}, J=14.7,9.4 \mathrm{~Hz}), 1.35(1 \mathrm{H}, \mathrm{dd}, J=14.7,1.7 \mathrm{~Hz}), 1.08(9 \mathrm{H}, \mathrm{s}), 0.90(3 \mathrm{H}$, 
s), $0.80(3 \mathrm{H}, \mathrm{s}) ;{ }^{13} \mathrm{C}$ NMR: $\delta$ 135.8, 135.7, 132.8, 132.7, 129.9, 129.8, 127.7, 127.7, 72.7, 68.6, 67.8, 44.0, 35.1, 26.9, 23.7, 19.3; MS (ESI) $(\mathrm{M}+\mathrm{Na})^{+}$calc'd for $\mathrm{C}_{23} \mathrm{H}_{34} \mathrm{O}_{3} \mathrm{SiNa}$ : 409.2. Found: 409.2.

Proof of Stereochemistry. Stereochemical ratios were determined in comparison to authentic racemic materials prepared by osmium tetraoxide catalyzed dihydroxylation. Absolute stereochemistry established in comparison to authentic (2R) isomer prepared via a Sharpless asymmetric dihydroxylation (Becker, H.; King, S. B.; Taniguchi, M.; Vanhessche, K.; Sharpless, K. B. J. Org. Chem. 1995, 60, 3940). 
Chiral HPLC (Chiralcel OD-H, Daicel, $0.1 \%$ iPrOH in hexanes, $1.0 \mathrm{~mL} / \mathrm{min}$, wavelength: $220 \mathrm{~nm}$ ) analysis of the acetonide (dimethoxypropane/cat. pTsOH) product:

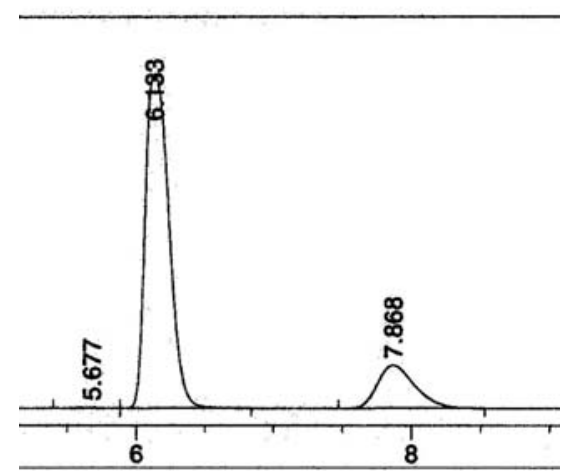

Diboration product

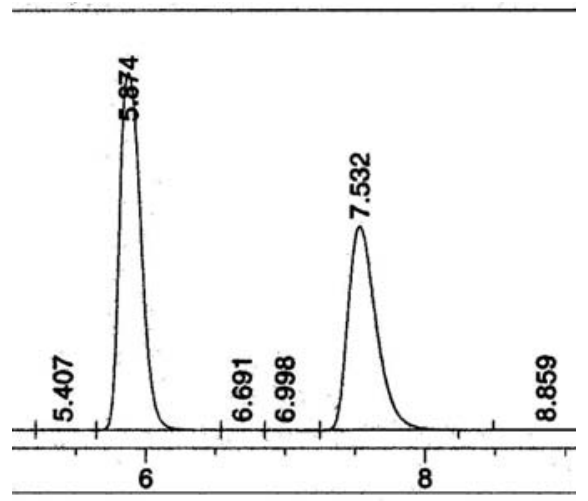

Authentic

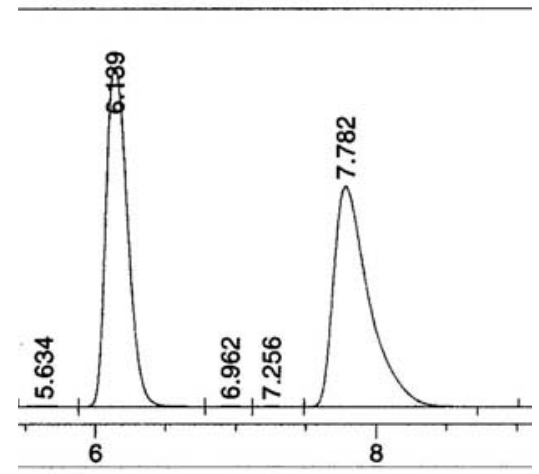

Racemic

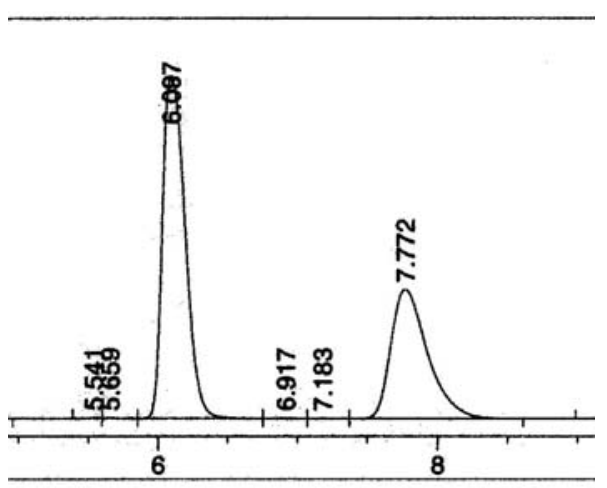

Racemic + Diboration product

Table 6, entry 2<smiles>CC(C)[C@@](C)(O)CO</smiles>

(2R)-2,3-dimethylbutane-1,2-diol. IR (neat, $v \mathrm{~cm}^{-1}$ ): 3398, 2964, 2879; ${ }^{1} \mathrm{H}$ NMR: $\delta 3.43$ (2H, dd, $J=48.7,11.1 \mathrm{~Hz}), 3.10(2 \mathrm{H}, \mathrm{br} \mathrm{s}), 1.77(1 \mathrm{H}, \mathrm{sp}, J=6.9 \mathrm{~Hz}), 1.00(3 \mathrm{H}, \mathrm{s}), 0.90$ $(3 \mathrm{H}, \mathrm{d}, J=6.9 \mathrm{~Hz}), 0.82(3 \mathrm{H}, \mathrm{d}, J=6.9 \mathrm{~Hz}) ;{ }^{13} \mathrm{C}$ NMR: $\delta 75.3,68.3,34.1,18.7,17.6$, 16.6; MS (ESI) $(\mathrm{M}+\mathrm{Na})^{+}$calc'd for $\mathrm{C}_{6} \mathrm{H}_{14} \mathrm{O}_{2} \mathrm{Na}$ : 141.1. Found: 141.1. 
Proof of Stereochemistry. Stereochemical ratios were determined in comparison to authentic racemic materials prepared by osmium tetraoxide catalyzed dihydroxylation. Absolute stereochemistry established in comparison to authentic $(2 R)$ isomer prepared via a Sharpless asymmetric dihydroxylation (Becker, H.; King, S. B.; Taniguchi, M.; Vanhessche, K.; Sharpless, K. B. J. Org. Chem. 1995, 60, 3940).

Chiral GLC ( $\beta$-dex, Supelco, $60^{\circ} \mathrm{C}, 20 \mathrm{psi}$ ) analysis of the acetonide (dimethoxypropane /cat. pTsOH) product:

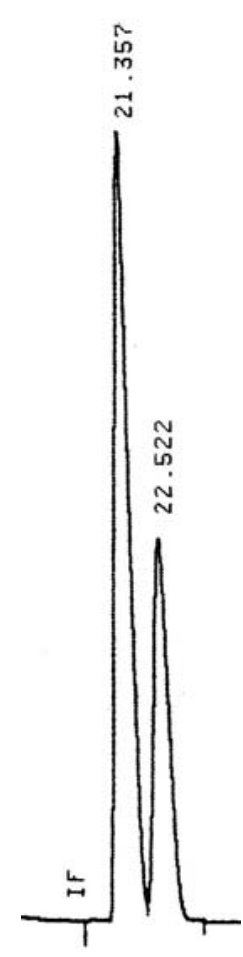

Diboration

product

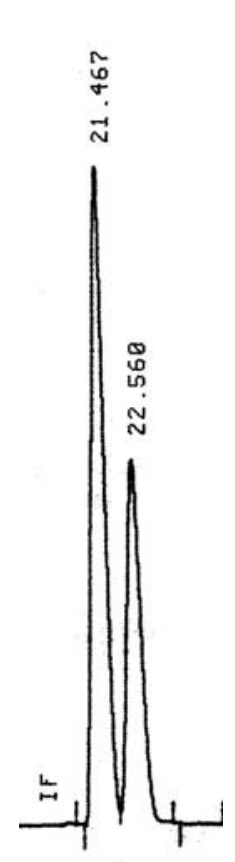

Authentic

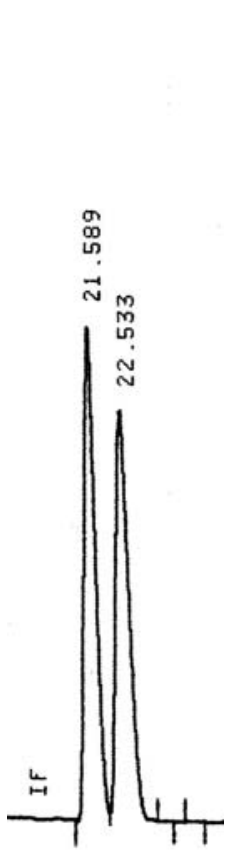

Racemic

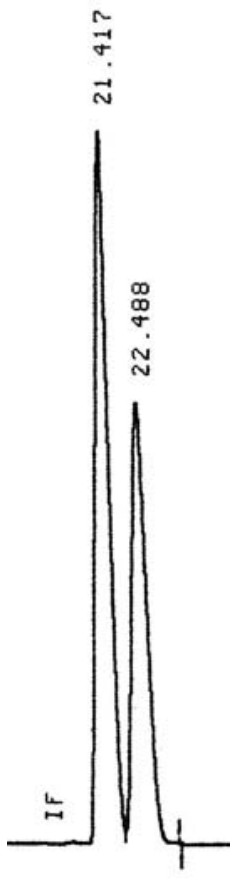

Racemic +

Diboration

Product 


\section{Table 6, entry 3}

$t$-butyl $\underbrace{\prime \prime \prime} \mathrm{OH}$

(2R)-2,4,4-trimethylpentane-1,2-diol. IR (neat, $v \mathrm{~cm}^{-1}$ ): 3398, 2964, 2879; ${ }^{1} \mathrm{H}$ NMR: $\delta$

$3.36(2 \mathrm{H}, \mathrm{dd}, J=41.0,10.8 \mathrm{~Hz}), 2.58(2 \mathrm{H}$, br s), $1.44(2 \mathrm{H}, \mathrm{dd}, J=48.2,14.8 \mathrm{~Hz}), 1.24$

$(3 \mathrm{H}, \mathrm{s}), 1.00(9 \mathrm{H}, \mathrm{s}) ;{ }^{13} \mathrm{C}$ NMR: $\delta 74.2,73.1,50.7,31.5,31.1,24.8$; HRMS (ESI)

$(\mathrm{M}+\mathrm{Na})^{+}$calc'd for $\mathrm{C}_{8} \mathrm{H}_{18} \mathrm{O}_{2} \mathrm{Na}$ : 169.1199. Found: 169.1202.

Proof of Stereochemistry. Stereochemical ratios were determined in comparison to authentic racemic materials prepared by osmium tetraoxide catalyzed dihydroxylation. Absolute stereochemistry established in comparison to authentic $(2 R)$ isomer prepared via a Sharpless asymmetric dihydroxylation (Becker, H.; King, S. B.; Taniguchi, M.; Vanhessche, K.; Sharpless, K. B. J. Org. Chem. 1995, 60, 3940). 
Chiral GLC ( $\beta$-dex, Supelco, $80^{\circ} \mathrm{C}, 20 \mathrm{psi}$ ) analysis of the acetonide (dimethoxypropane /cat. pTsOH) product:

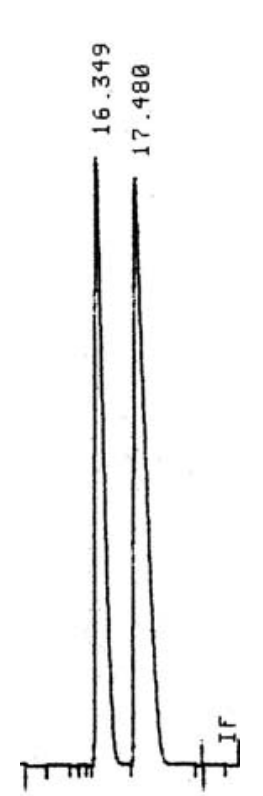

Diboration

product

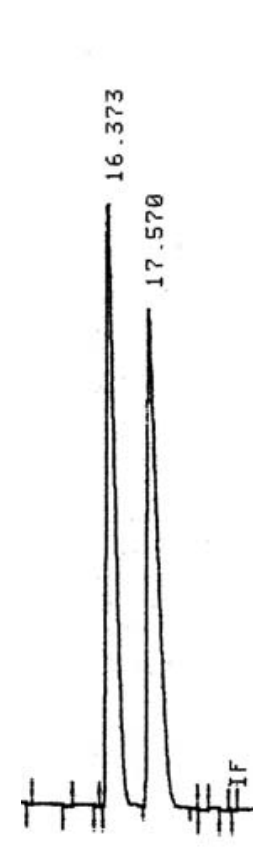

Racemic

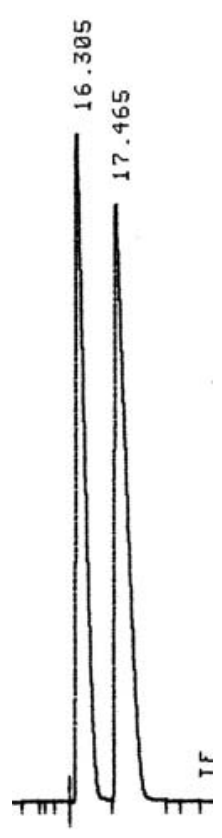

Racemic +

Diboration

product

\section{Table 6, entry 6}<smiles>CCCCC(O)C(C)(C)O</smiles>

(3R)-2-methyl-heptane-2,3-diol. IR (neat, $v \mathrm{~cm}^{-1}$ ): 3394, 2958, 2933, 2873, 2861, 1466, 1380, 1167, 1071; ${ }^{1} \mathrm{H}$ NMR: $\delta 3.35$ (1H, dd, $\left.J=10.1,2.3 \mathrm{~Hz}\right), 1.66-1.22(8 \mathrm{H}, \mathrm{m}), 1.19$ (3H, s), 1.14 (3H, s), 0.890 (3H, t, $J=7.3 \mathrm{~Hz}) ;{ }^{13} \mathrm{C}$ NMR: $\delta$ 78.6, 73.1, 31.4, 28.9, 26.5, 23.1, 22.7, 14.0; $\mathrm{MS}(\mathrm{ESI})(\mathrm{M}+\mathrm{Na})^{+}$calc'd for $\mathrm{C}_{8} \mathrm{H}_{18} \mathrm{O}_{2} \mathrm{Na}$ : 169.1. Found: 169.1 . 
Proof of Stereochemistry. Stereochemical ratios were determined in comparison to authentic racemic materials prepared by osmium tetraoxide catalyzed dihydroxylation.

Chiral GLC ( $\beta$-dex, Supelco, $\left.130^{\circ} \mathrm{C}, 20 \mathrm{psi}\right)$ analysis of the product:

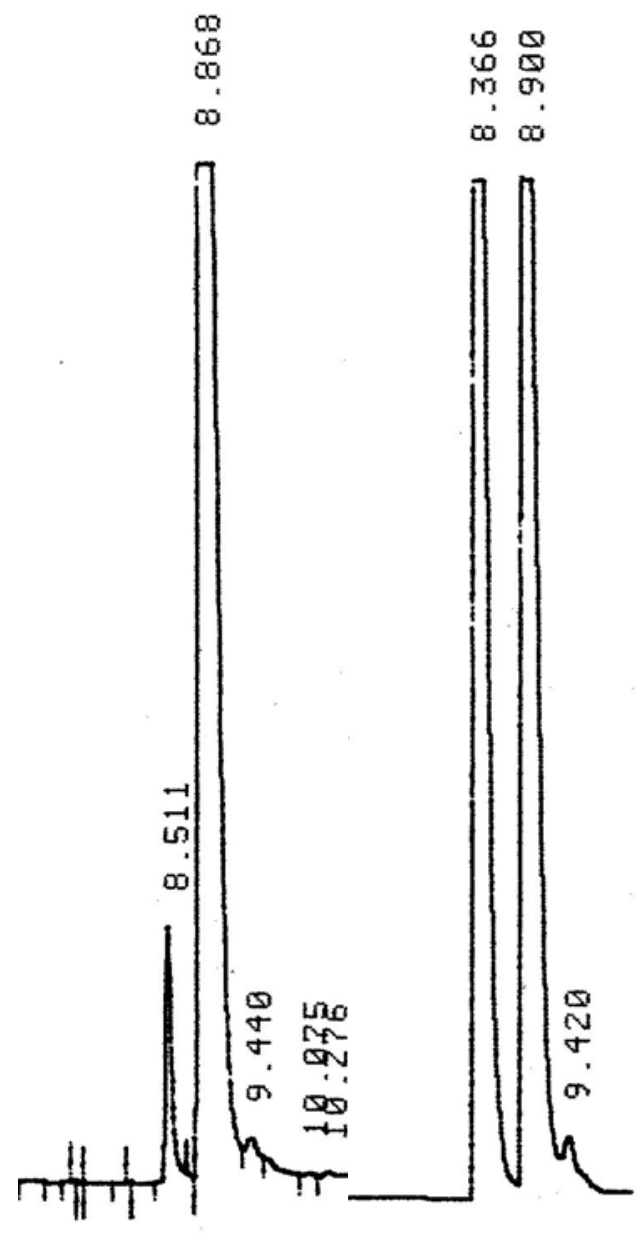

Diboration Racemic

product 


\section{Scheme 3 (substrate)}

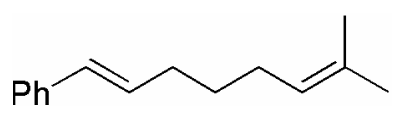

trans-(7-methyl-octa-1,6-dienyl)-benzene. To a stirred suspension of isopropyl(triphenyl)phosphonium bromide $(1.28 \mathrm{~g}, 3.33 \mathrm{mmol})$ in $\mathrm{THF}(6.0 \mathrm{~mL})$ at $0^{\circ} \mathrm{C}$ was added a solution of (E)-6-phenyl-hex-5-enal ${ }^{3}(0.29 \mathrm{~g}, 1.66 \mathrm{mmol})$ in THF $(5.0 \mathrm{~mL})$. The resulting mixture was stirred $0.5 \mathrm{~h}$ at $0^{\circ} \mathrm{C}$ and $0.5 \mathrm{~h}$ at room temperature. Water was then added and mixture was extracted three times with $\mathrm{Et}_{2} \mathrm{O}$. The combined organic phases were dried with $\mathrm{MgSO}_{4}$, filtered and concentrated under reduced pressure. The crude product was purified by flash chromatography (100/0 to 80/0: hex/EtOAc) to yield the product $(0.17 \mathrm{~g}, 50 \%)$ as a clear oil. IR (neat, $\left.v \mathrm{~cm}^{-1}\right): 2927,2856,1449 ;{ }^{1} \mathrm{H}$ NMR: $\delta$ 7.36-7.17 (5H, m), $6.39(1 \mathrm{H}, \mathrm{d}, J=15.8 \mathrm{~Hz}), 6.22(1 \mathrm{H}, \mathrm{dt}, J=15.8,6.9 \mathrm{~Hz}), 5.17-5.13$ $(1 \mathrm{H}, \mathrm{m}), 2.22(2 \mathrm{H}, \mathrm{q}, J=6.9 \mathrm{~Hz}), 2.04(2 \mathrm{H}, \mathrm{q}, J=7.4 \mathrm{~Hz}), 1.71(3 \mathrm{H}, \mathrm{s}), 1.62(3 \mathrm{H}, \mathrm{s})$, $1.52(2 \mathrm{H}, \mathrm{qu}, J=7.3 \mathrm{~Hz}) ;{ }^{13} \mathrm{C}$ NMR: $\delta 138.0,131.6,131.0,129.9,128.4,126.7,125.9$, $124.4,32.6,29.5,27.6,25.7,17.7$.

\section{Scheme 3 (product)}<smiles>CC(C)=CCCC[C@@H](O)[C@H](O)c1ccccc1</smiles>

(1R, 2R)-7-methyl-1-phenyl-oct-6-ene-1,2-diol. IR (neat, $v \mathrm{~cm}^{-1}$ ): 3375, 2927, 2860, 1455, 1081, 1054, 1025; ${ }^{1} \mathrm{H}$ NMR: $\delta$ 7.35-7.25 (5H, m), 5.03-4.98 (1H, m), $4.38(1 \mathrm{H}, \mathrm{d}, J$ $=6.8 \mathrm{~Hz}), 3.66-3.60(1 \mathrm{H}, \mathrm{m}), 2.78(2 \mathrm{H}, \mathrm{br} \mathrm{s}), 1.92-1.85(2 \mathrm{H}, \mathrm{m}), 1.62(3 \mathrm{H}, \mathrm{s}), 1.52(3 \mathrm{H}$, s), 1.51-1.25 (4H, m); ${ }^{13} \mathrm{C}$ NMR: $\delta 141.3,131.6,128.4,128.0,126.8,124.3,77.8,75.9$, 
32.3, 27.8, 25.8, 25.6, 17.6; MS (ESI) $(\mathrm{M}+\mathrm{Na})^{+}$calc'd for $\mathrm{C}_{15} \mathrm{H}_{22} \mathrm{O}_{2} \mathrm{Na}$ : 256.9. Found: 256.9 .

Proof of Stereochemistry. Stereochemical ratios were determined in comparison to authentic racemic materials prepared by diboration with racemic QUINAP followed by oxidation.

Chiral HPLC (Chiralcel OD-H, Daicel, $0.1 \% \mathrm{iPrOH}$ in hexanes, $1.0 \mathrm{~mL} / \mathrm{min}$, wavelength: $220 \mathrm{~nm}$ ) analysis of the acetonide (dimethoxypropane/cat. pTsOH) product:

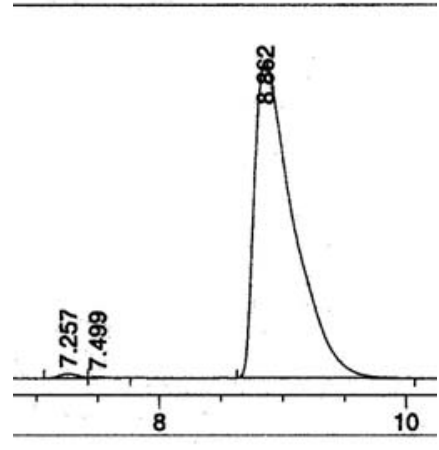

Diboration product

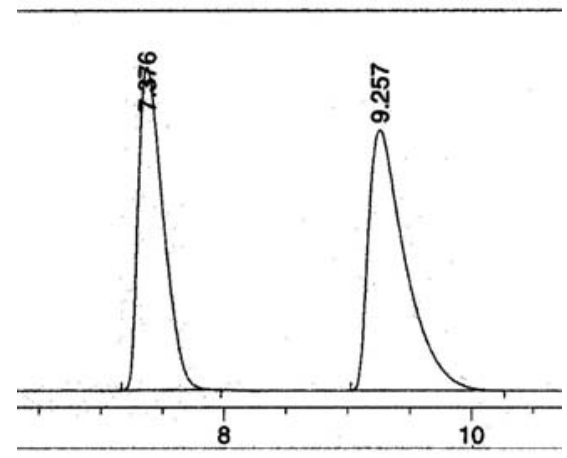

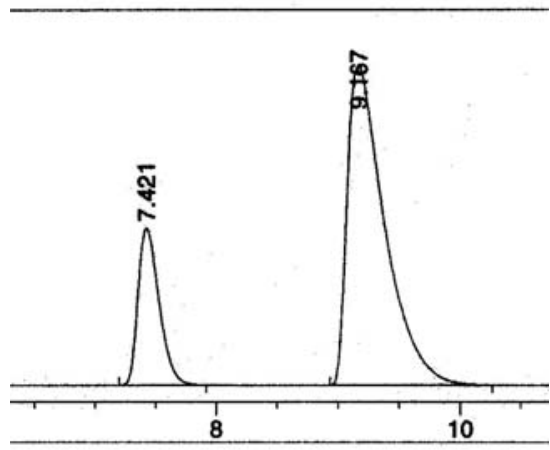

Racemic + Diboration product

Racemic 
3) ${ }^{1} \mathrm{H}$ and ${ }^{13} \mathrm{C}$ NMR spectra

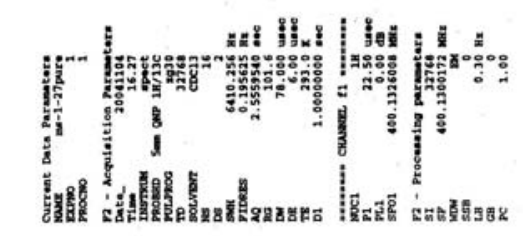

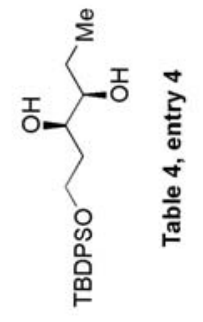

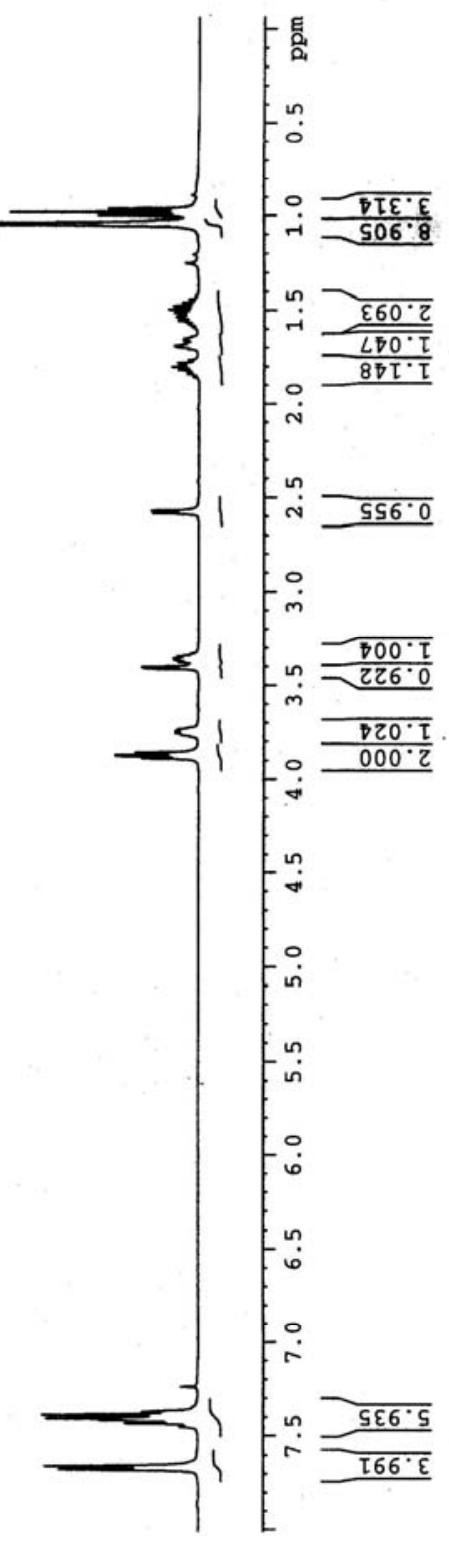

Page S22 

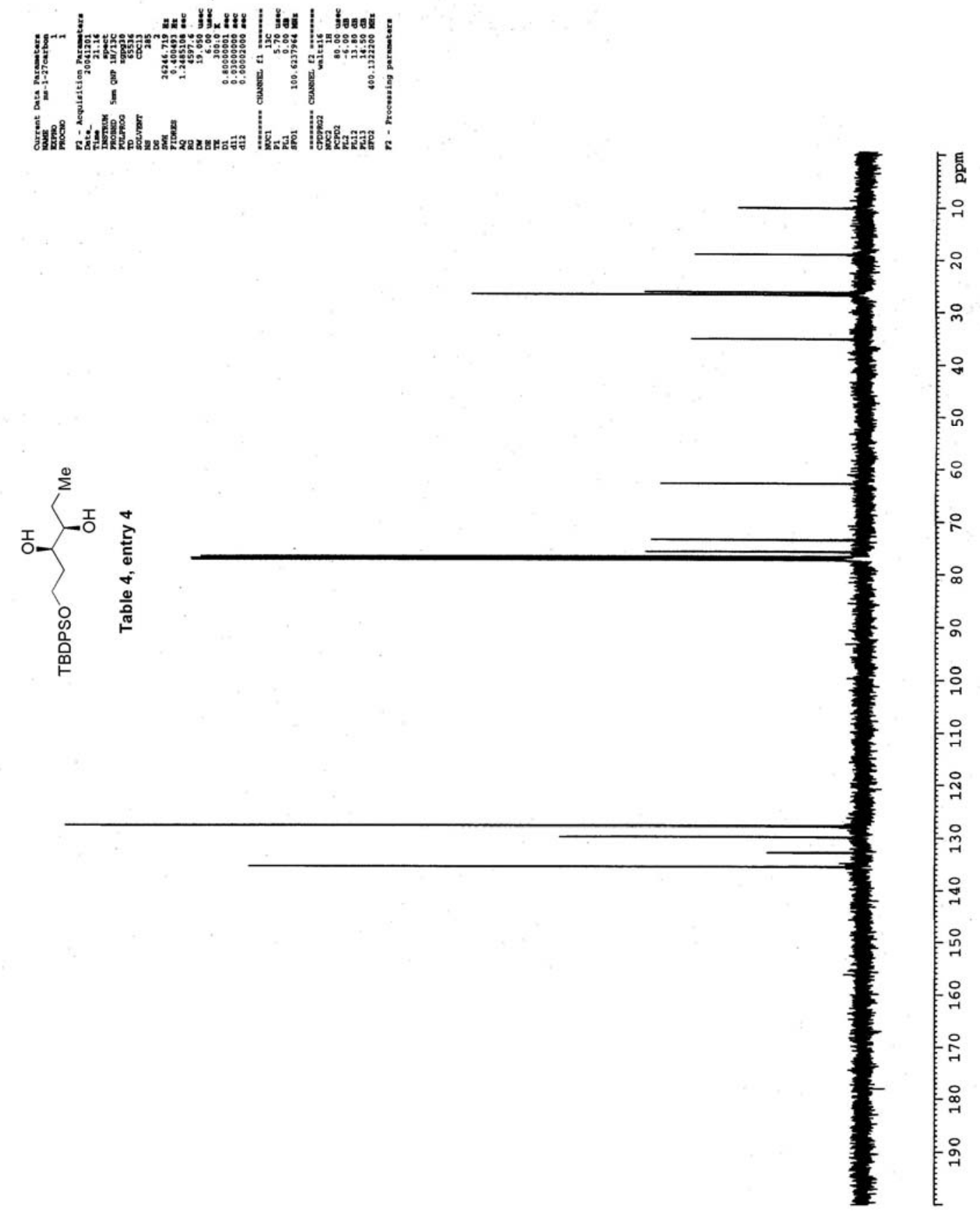

Page S23 


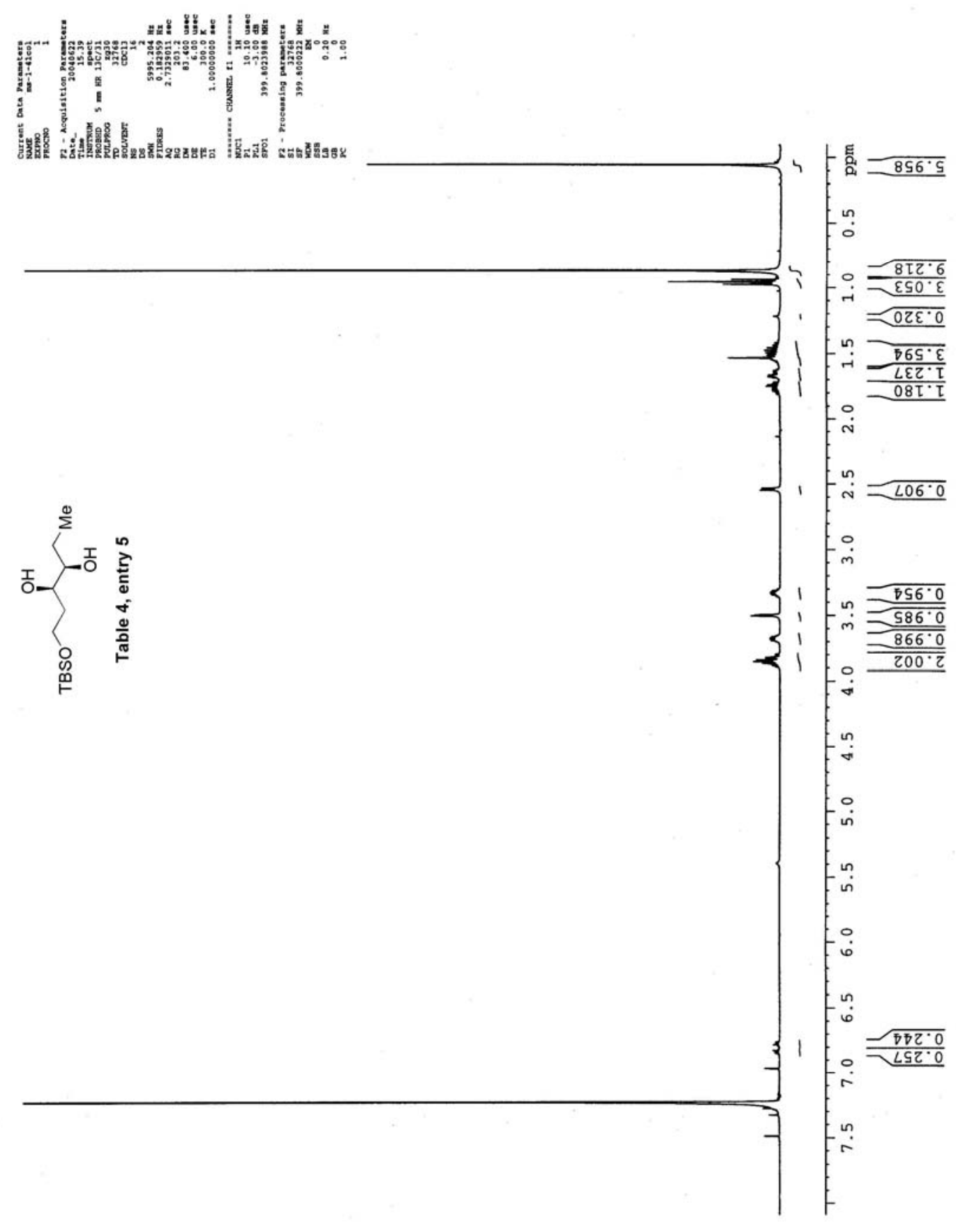




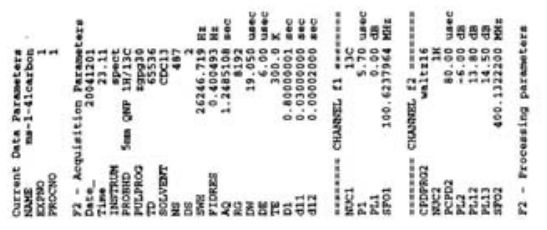

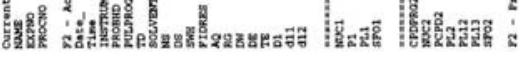

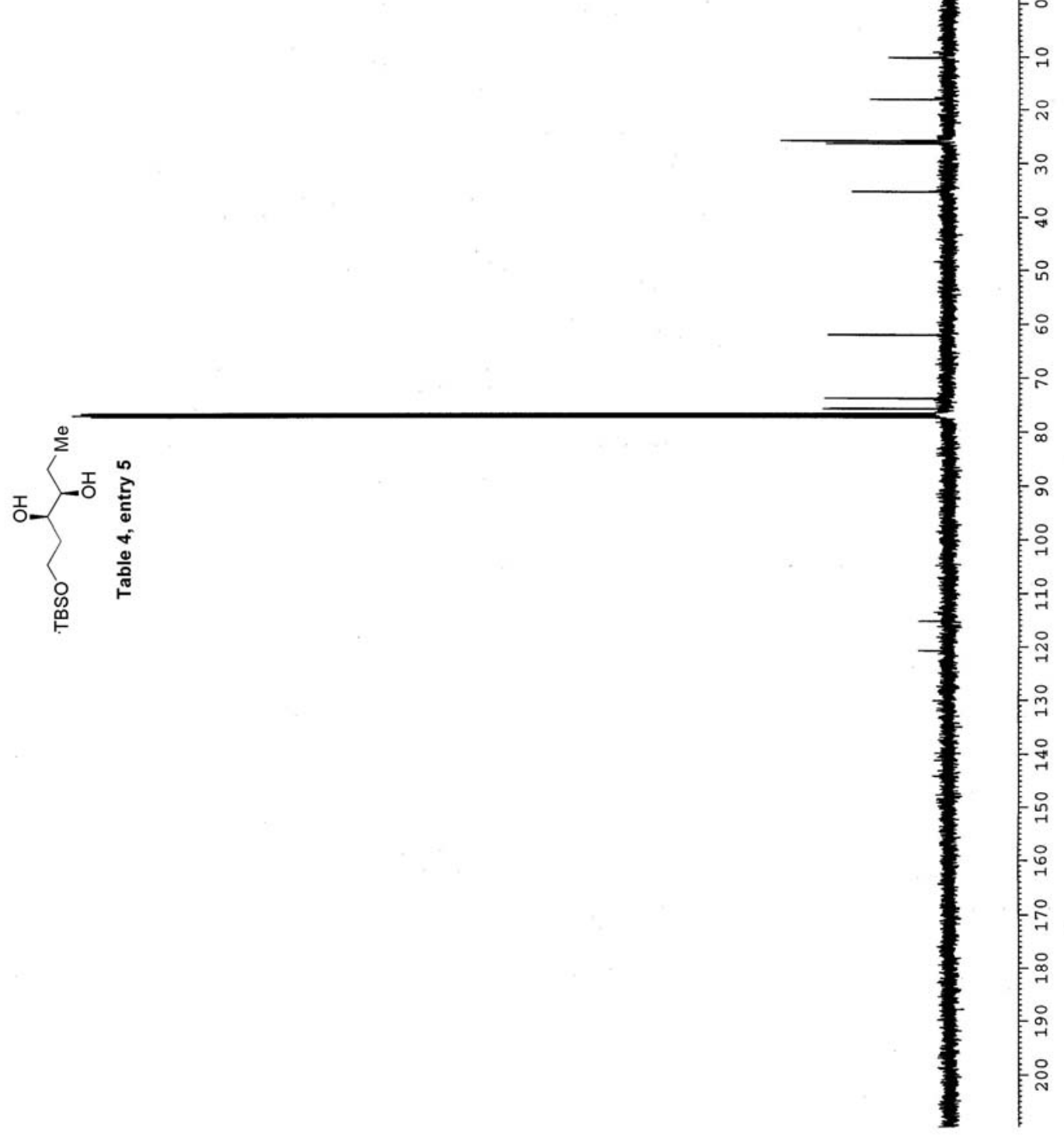

Page S25 

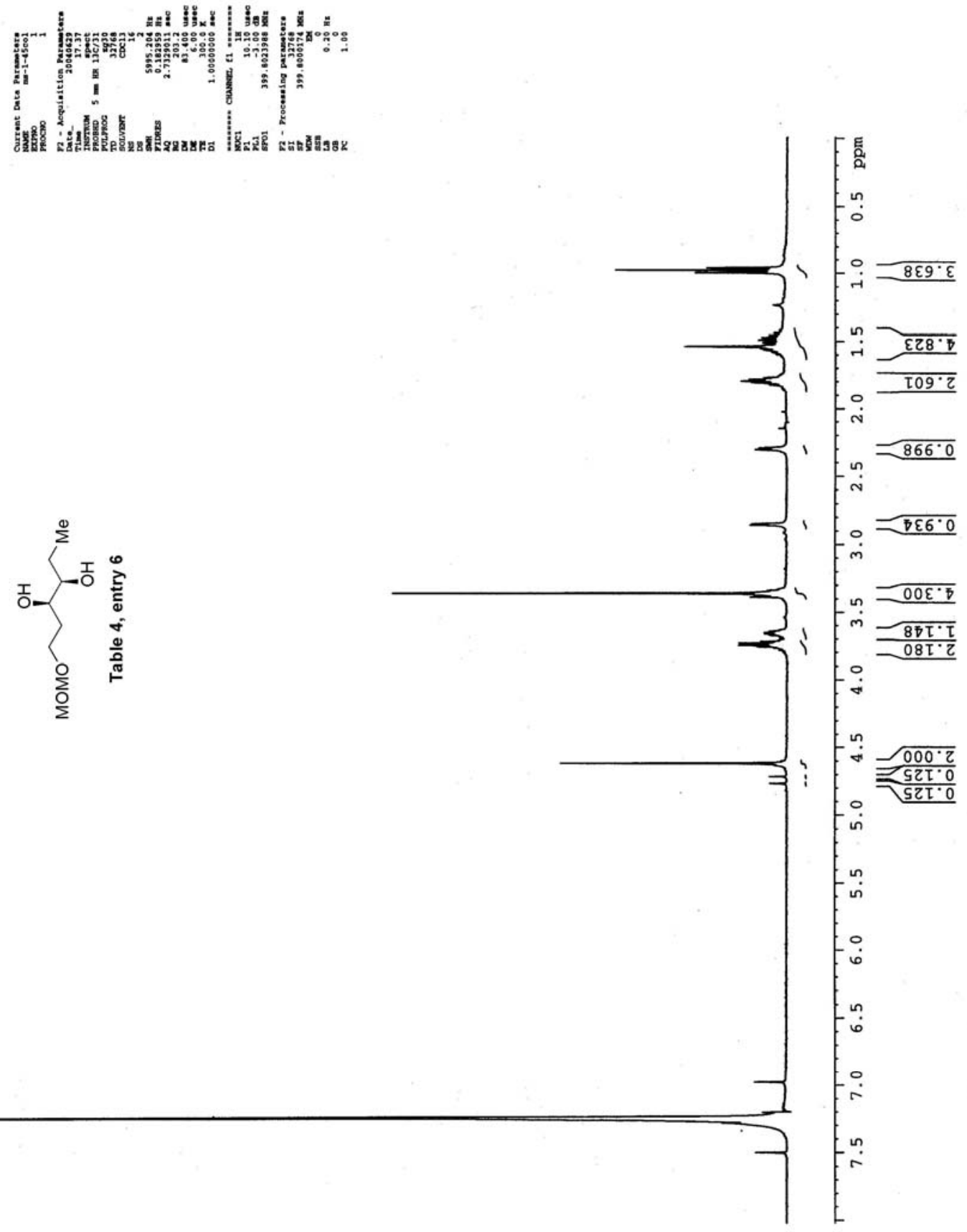

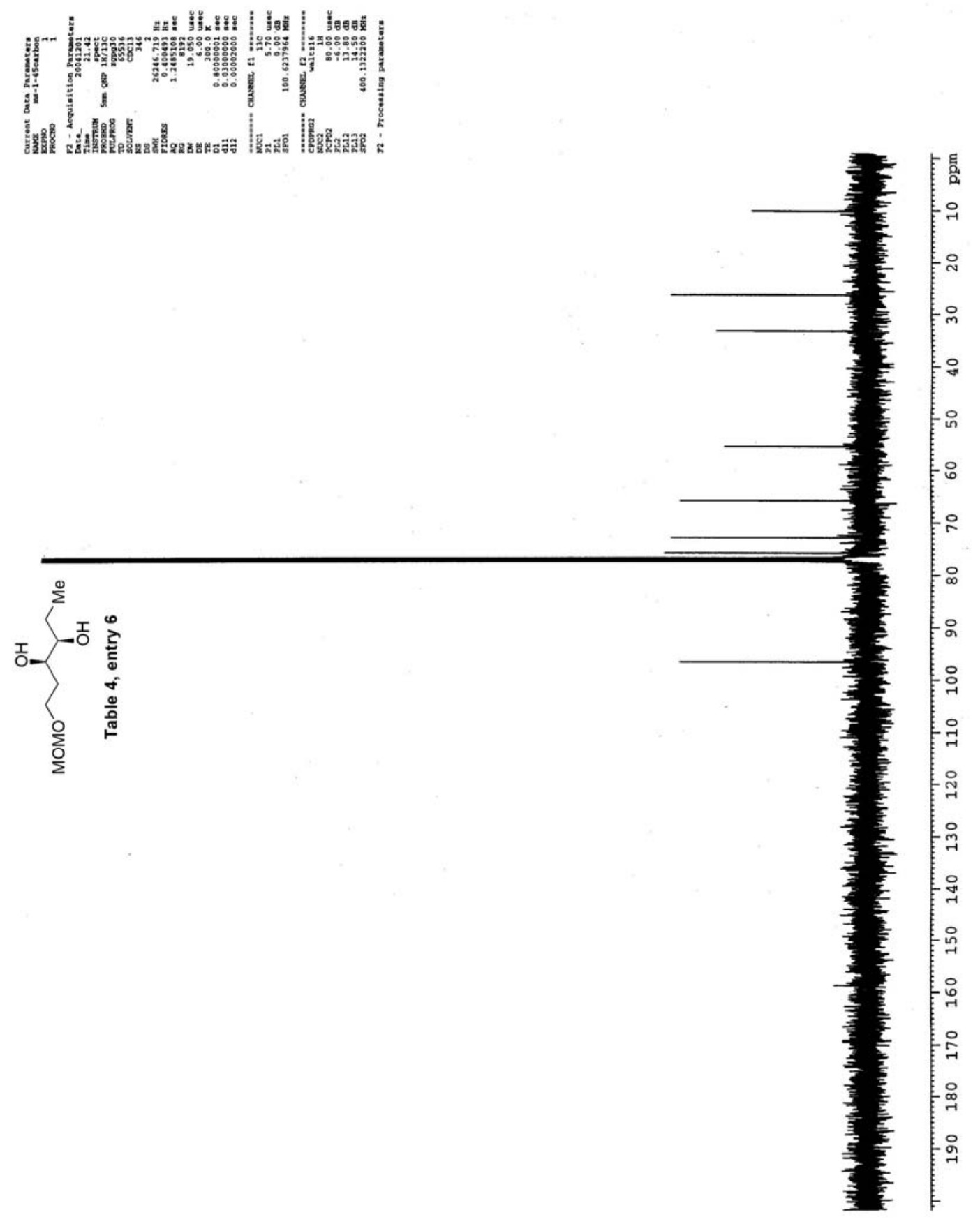

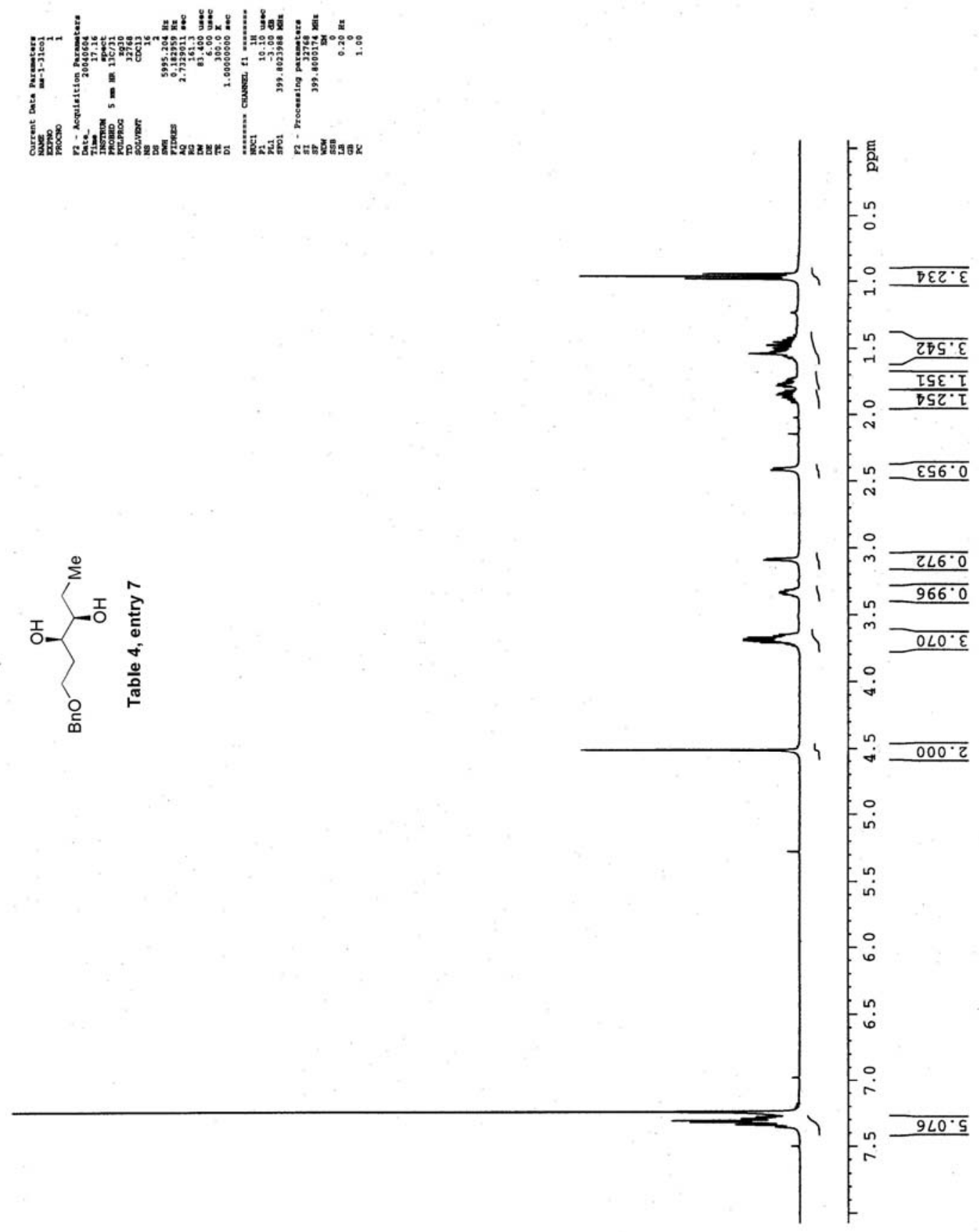

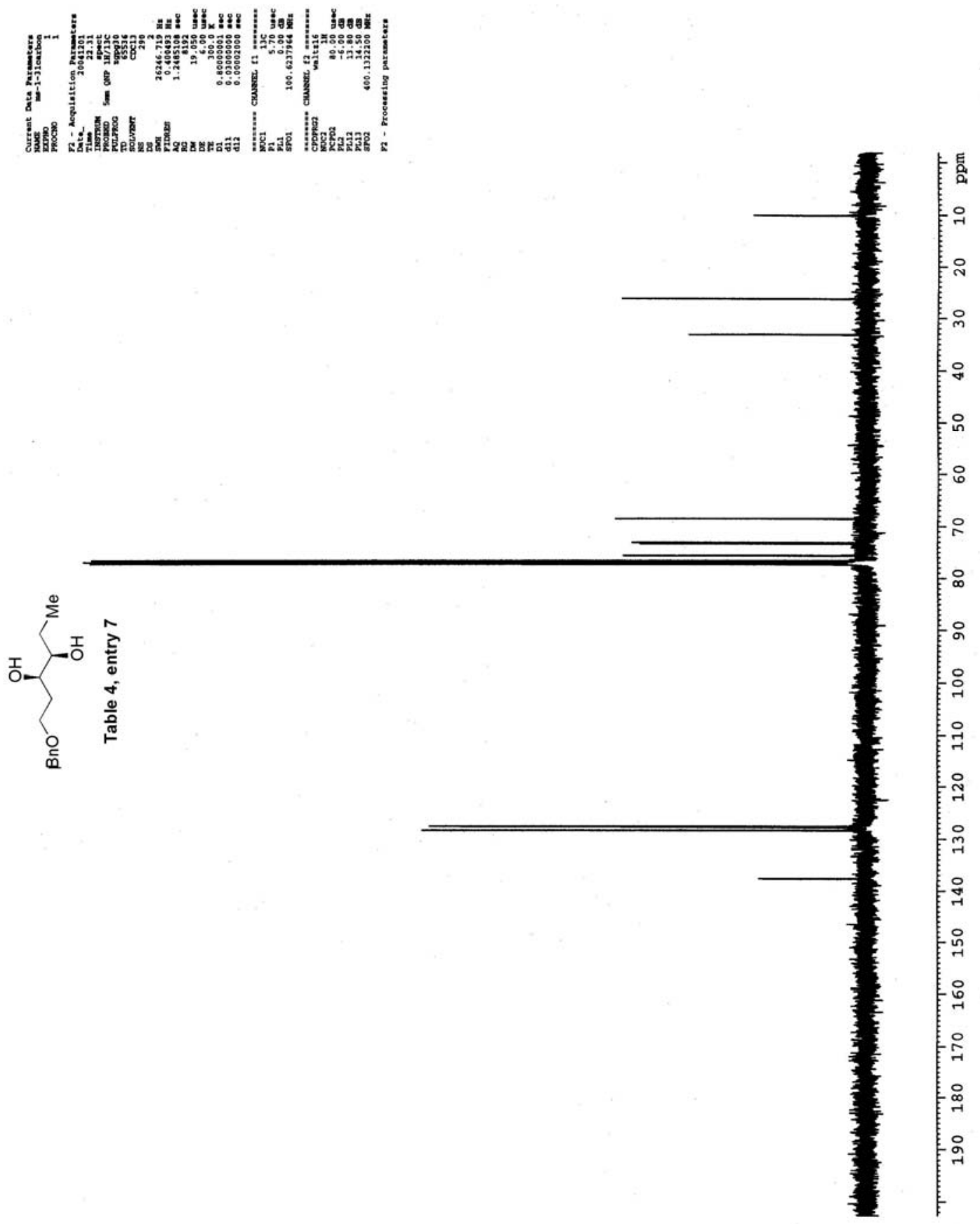

Page S29 


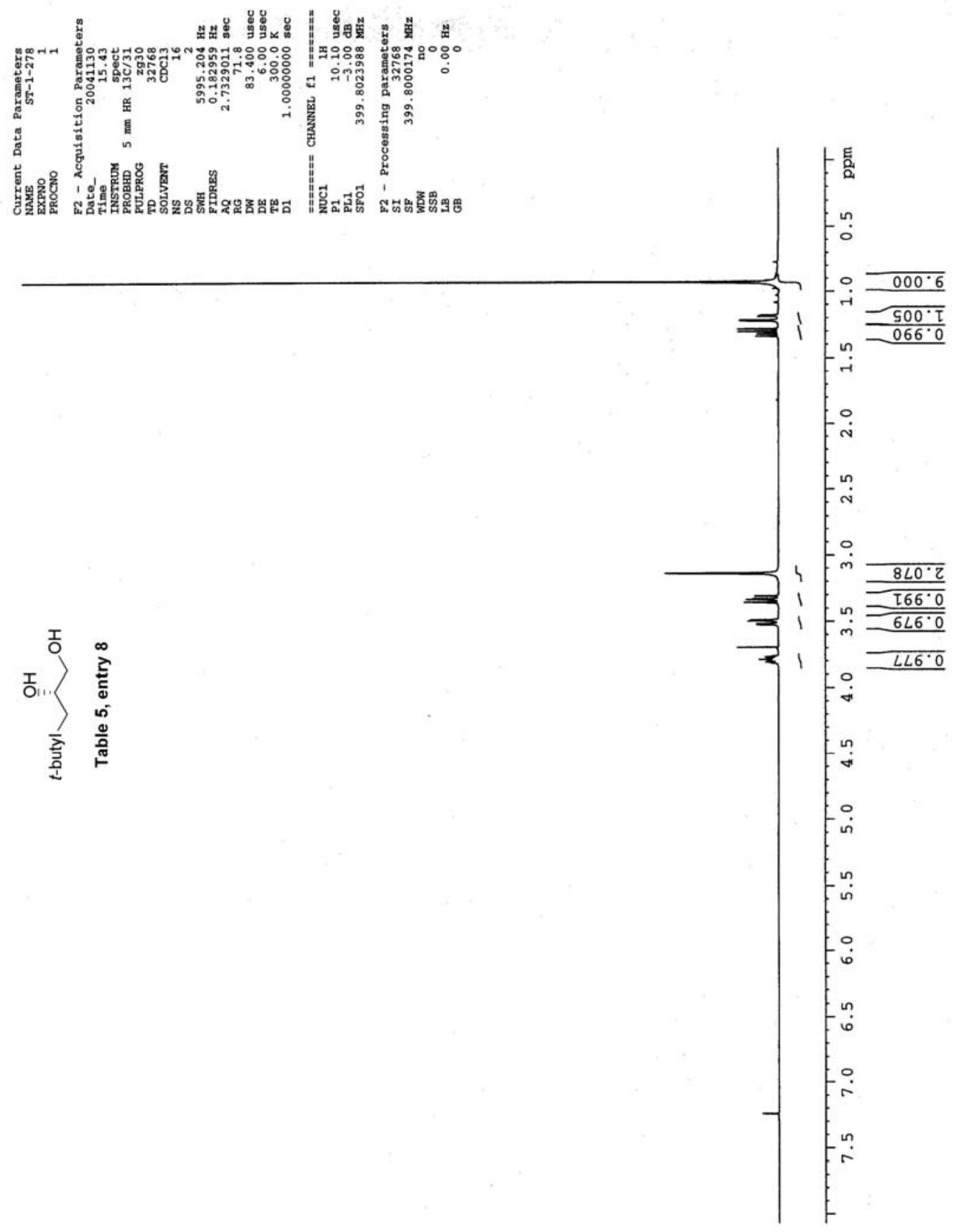

Page S30 


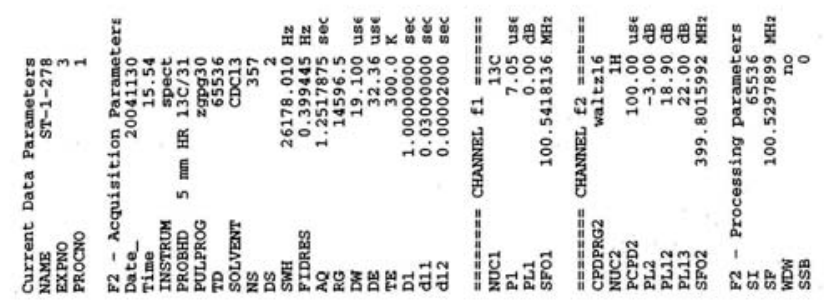

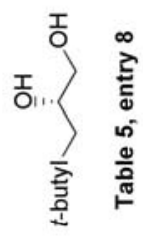

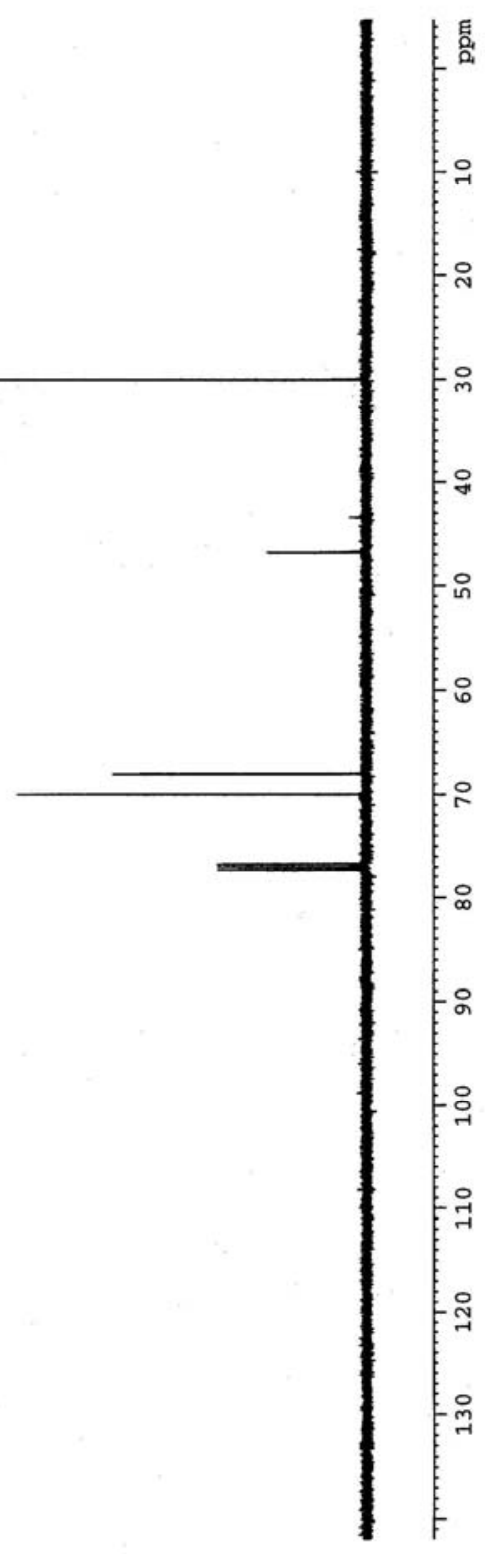




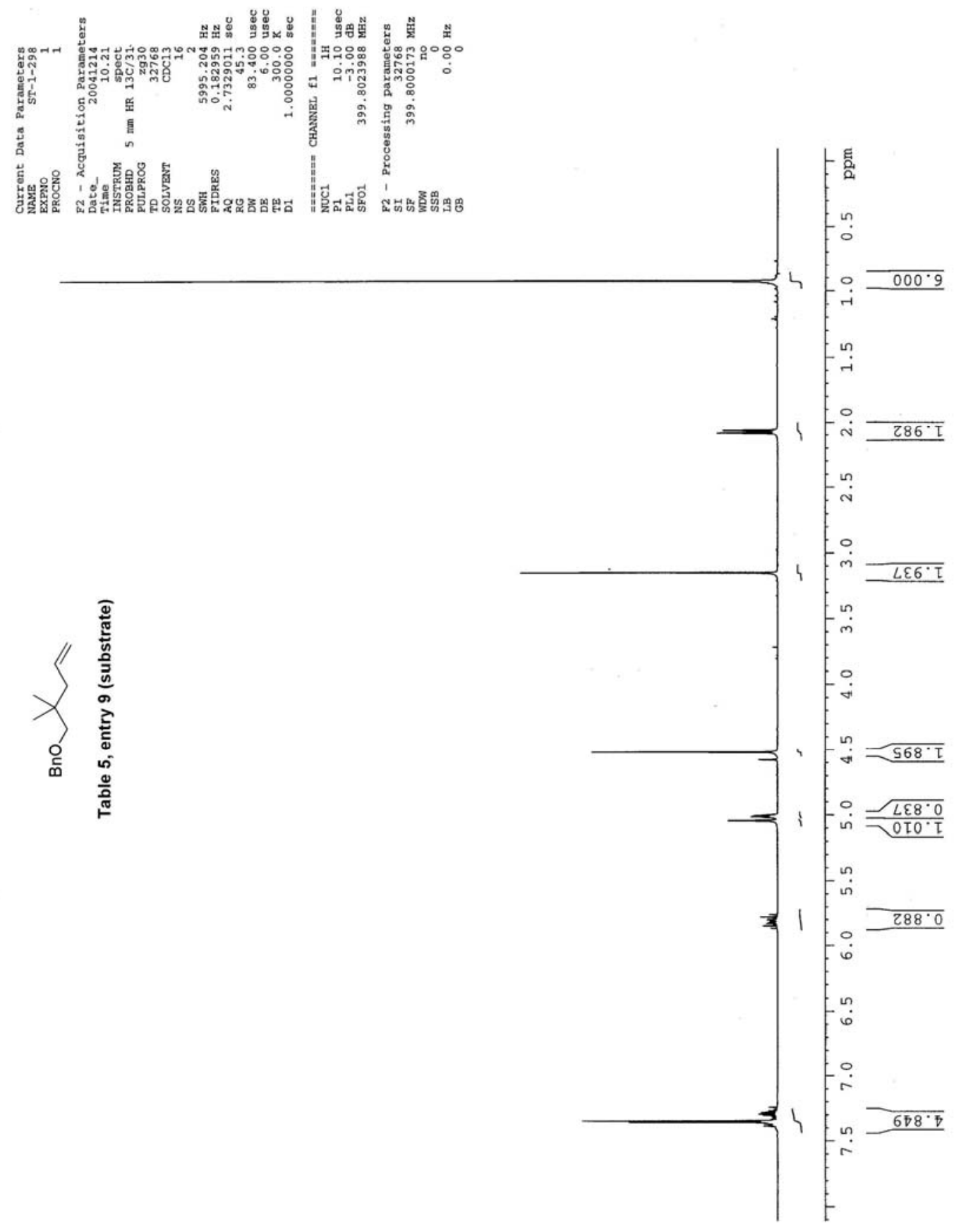

Page S32 


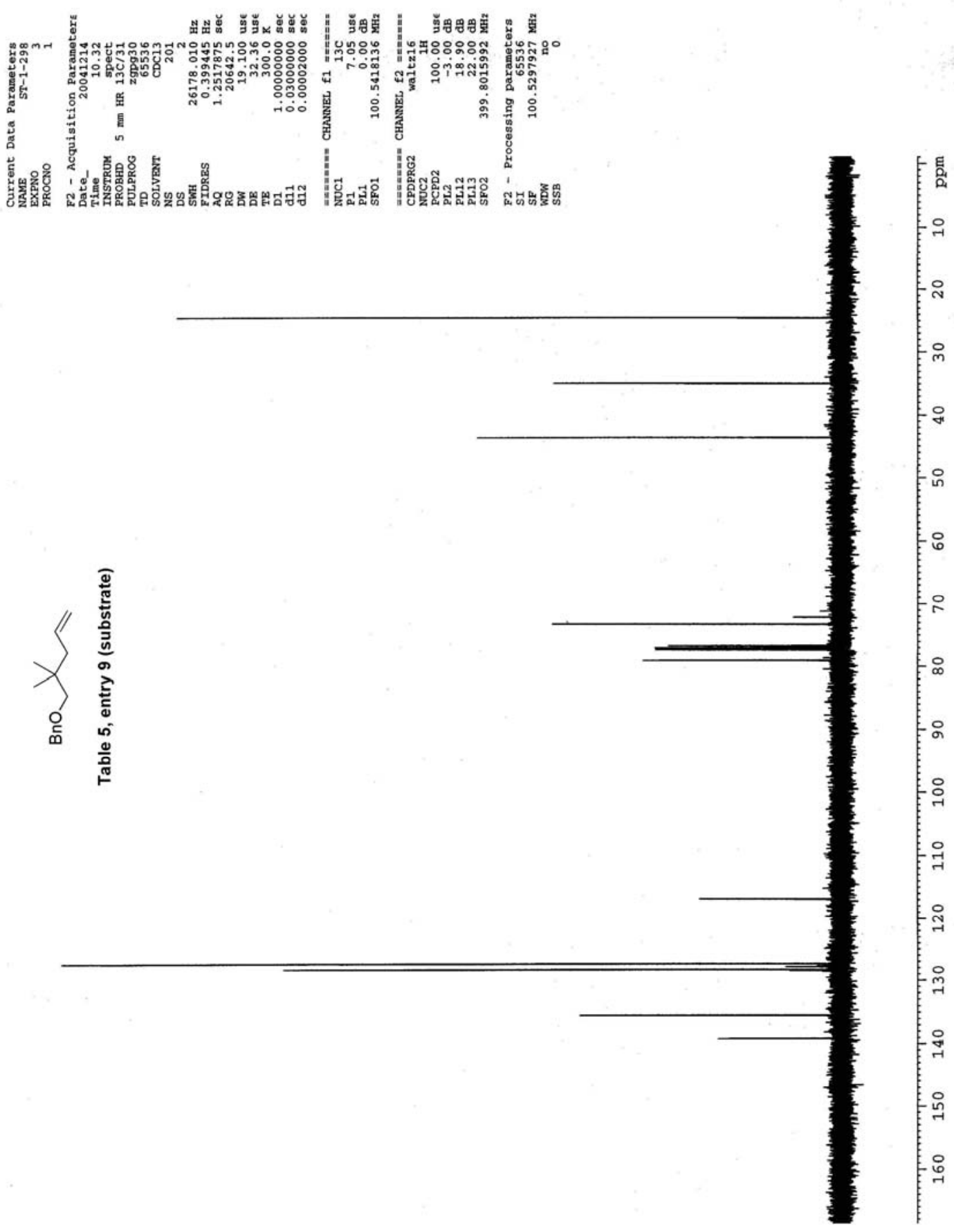

Page S33 


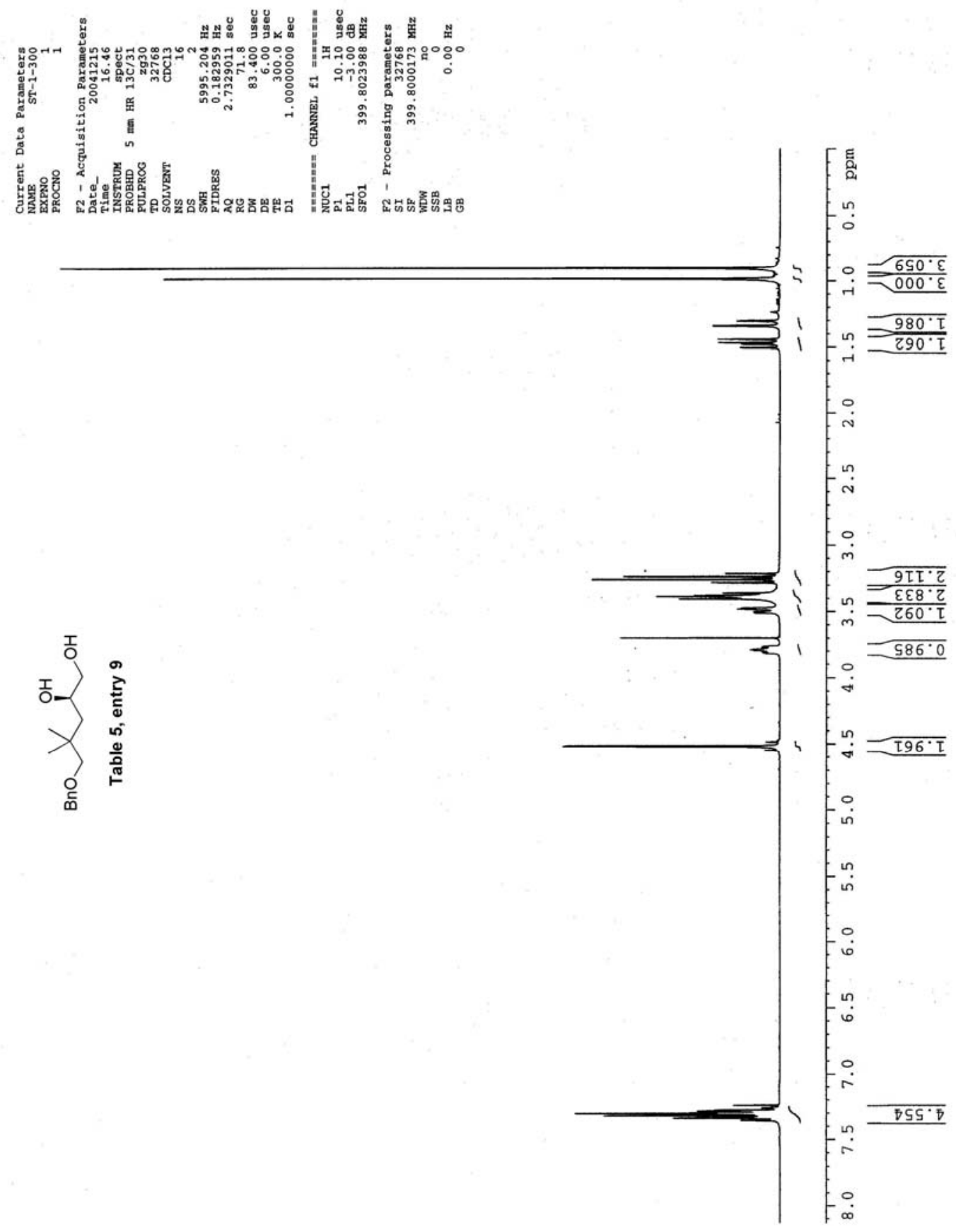




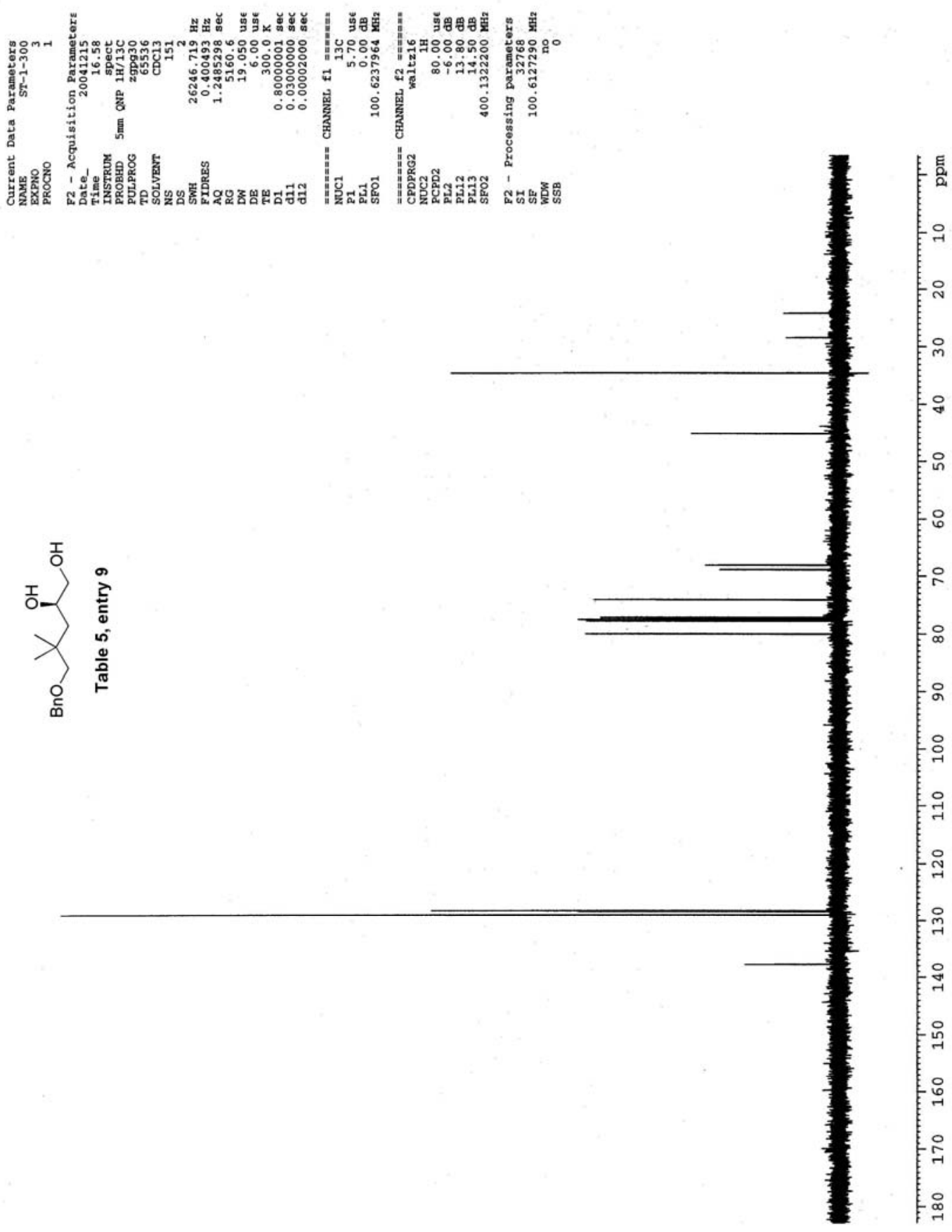

Page S35 


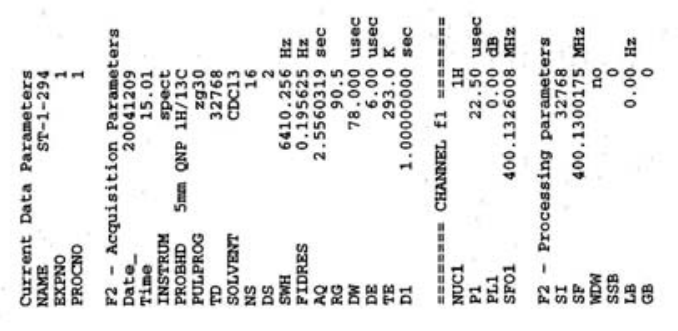

>->

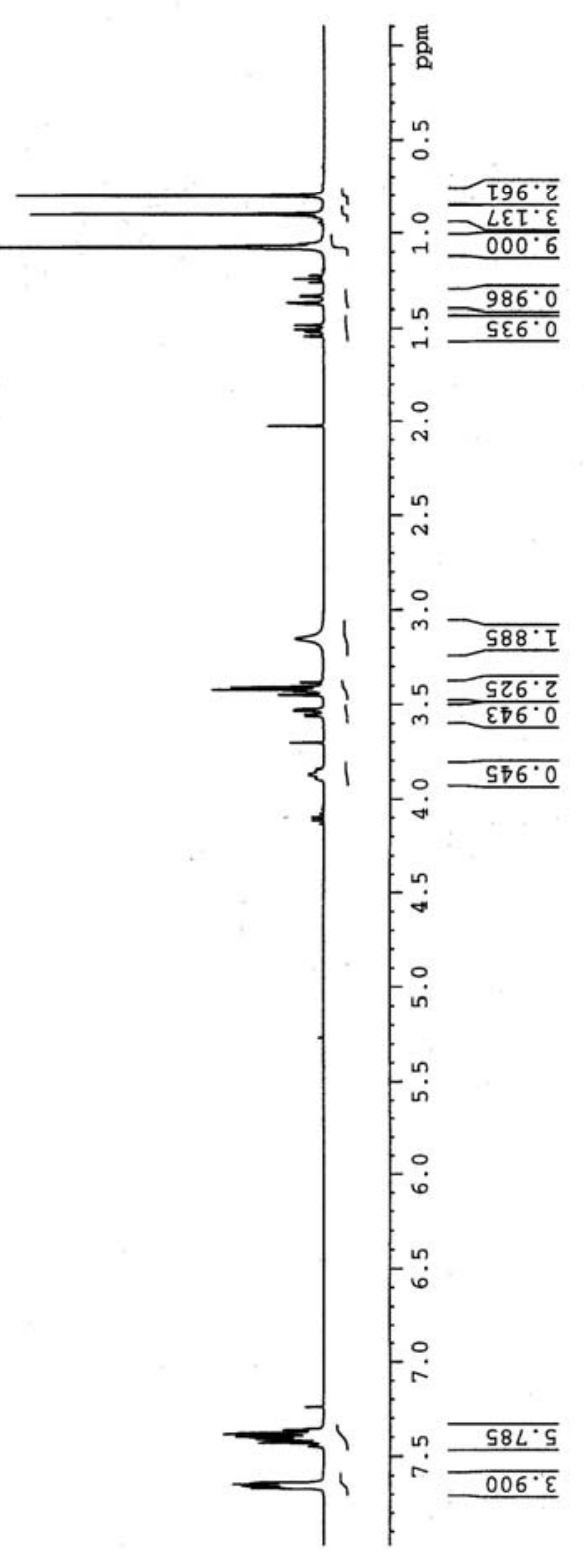

Page S36 


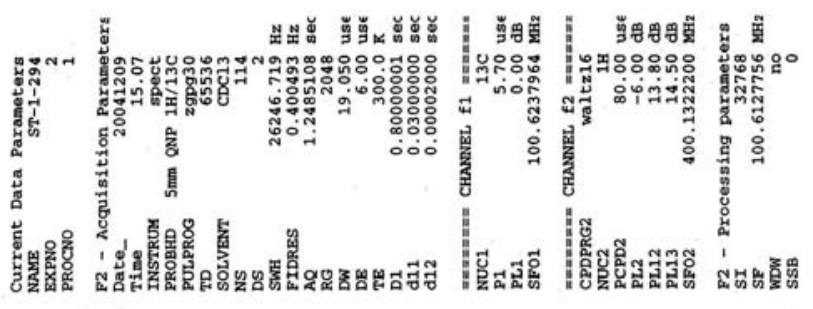

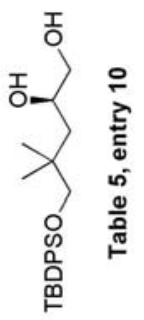

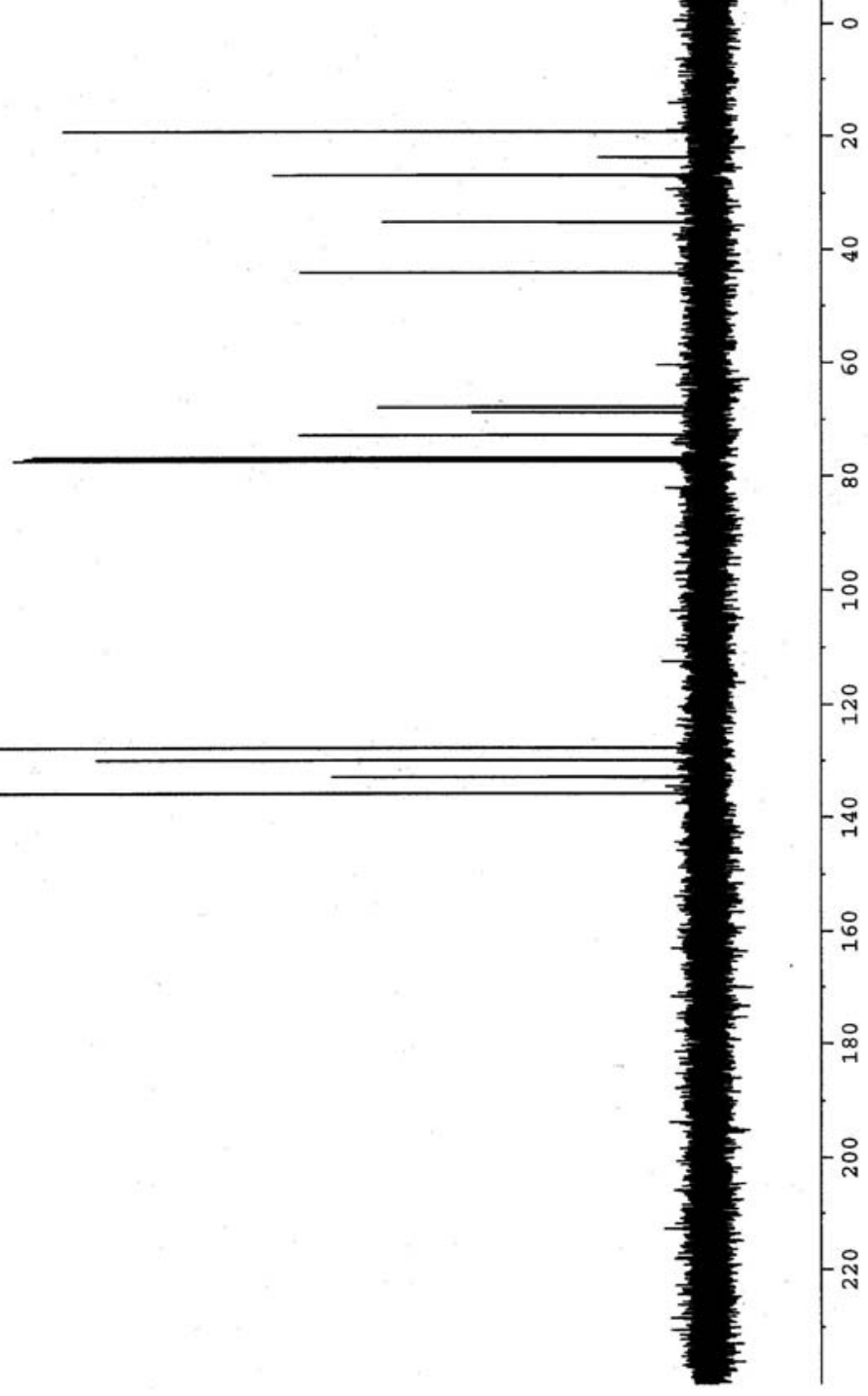




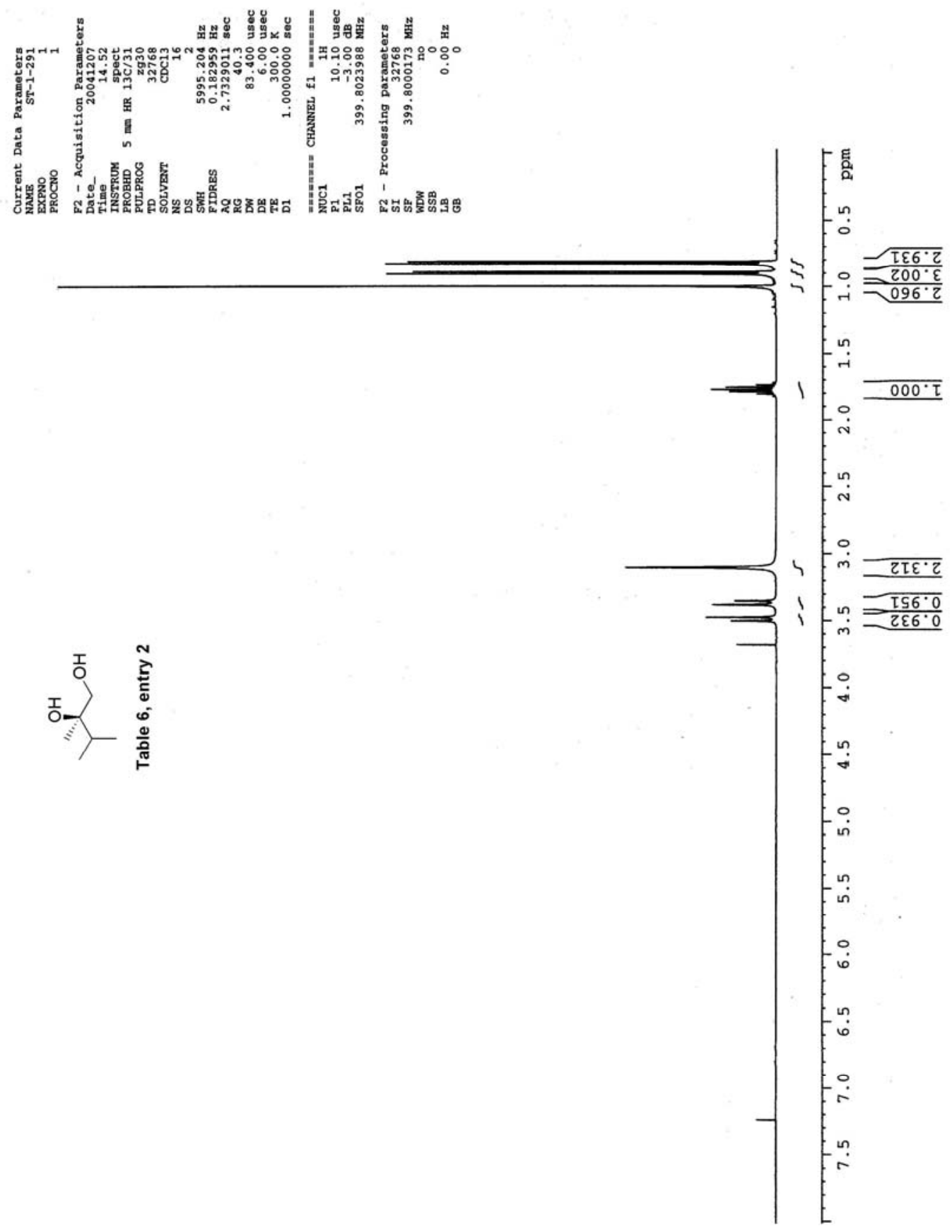

Page S38 


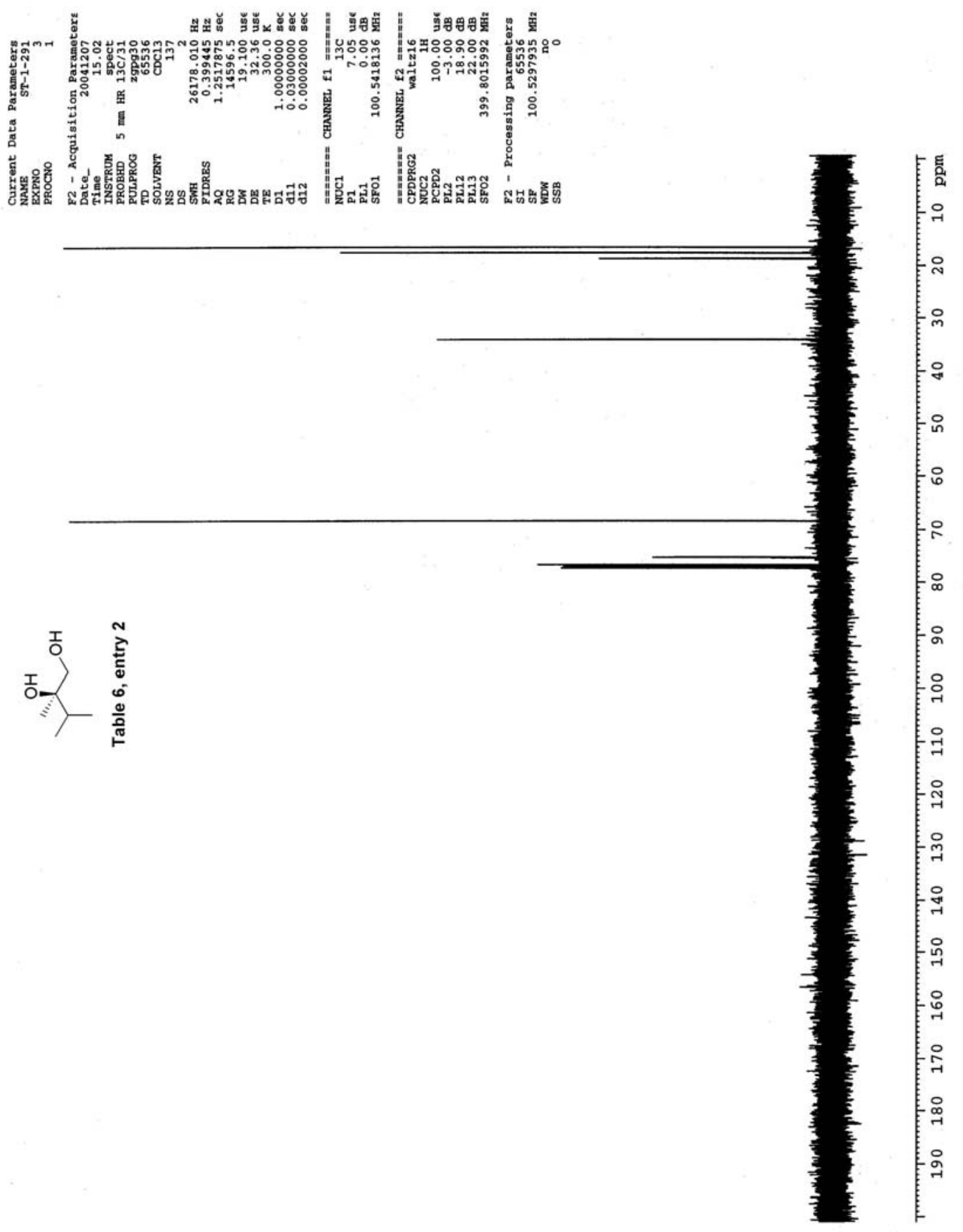

Page S39 


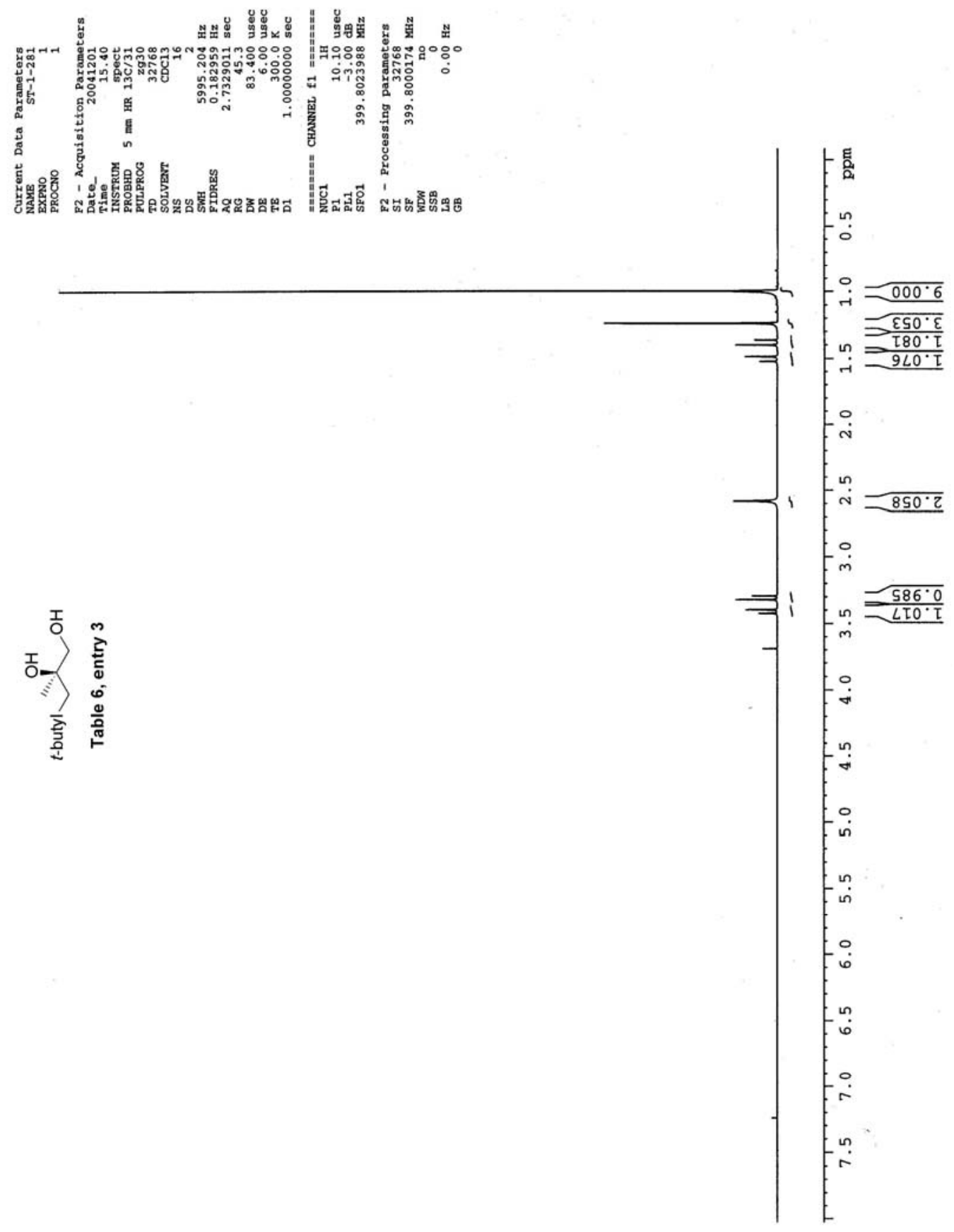

Page S40 


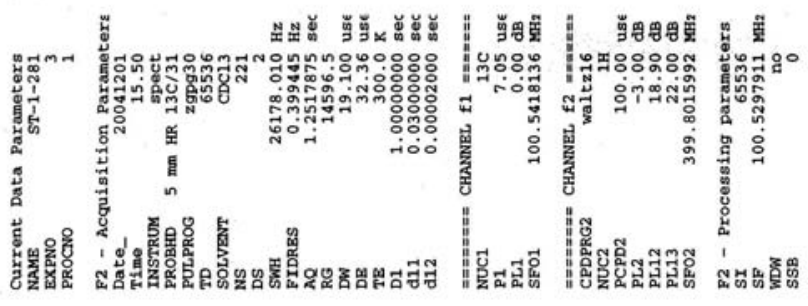

I

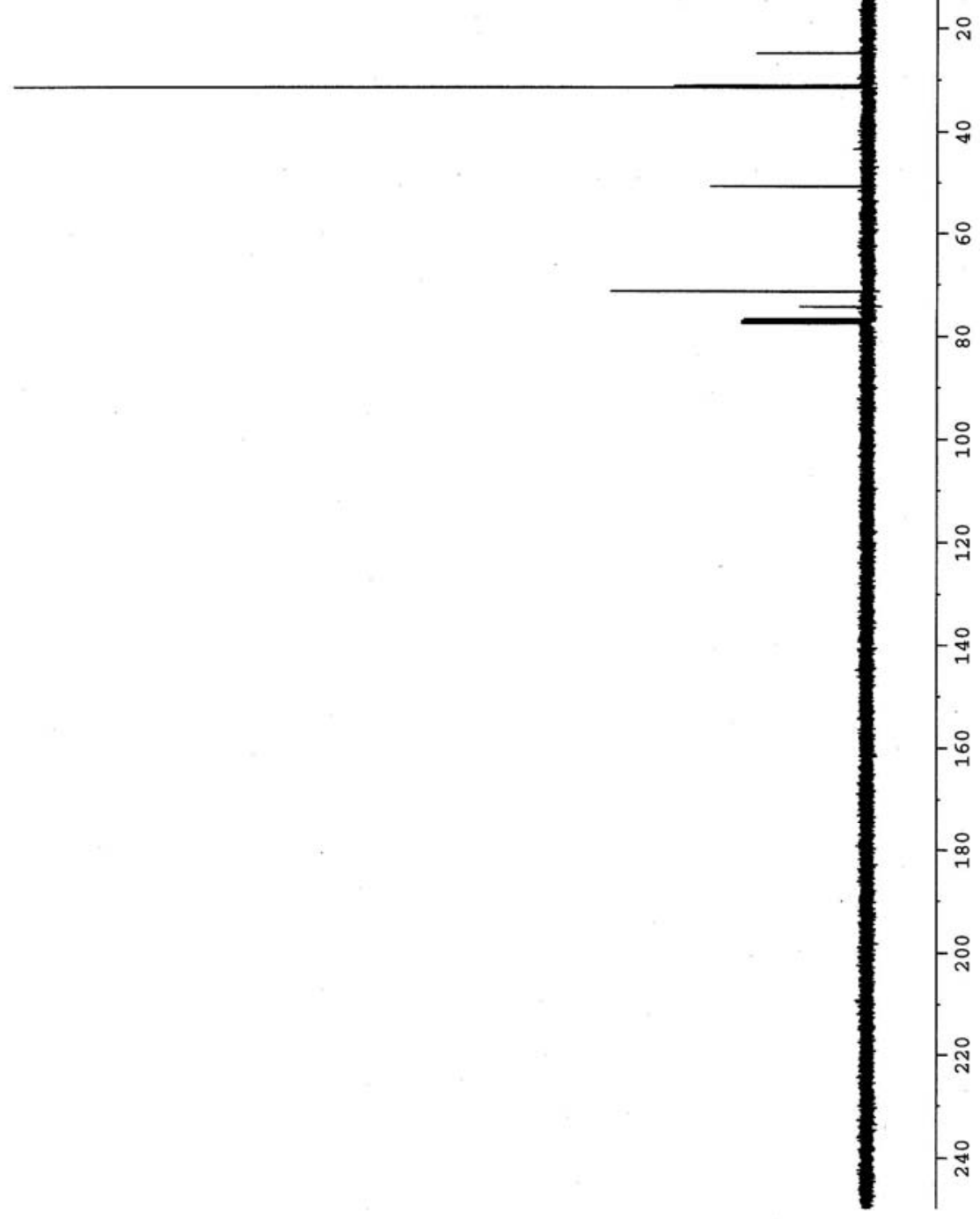




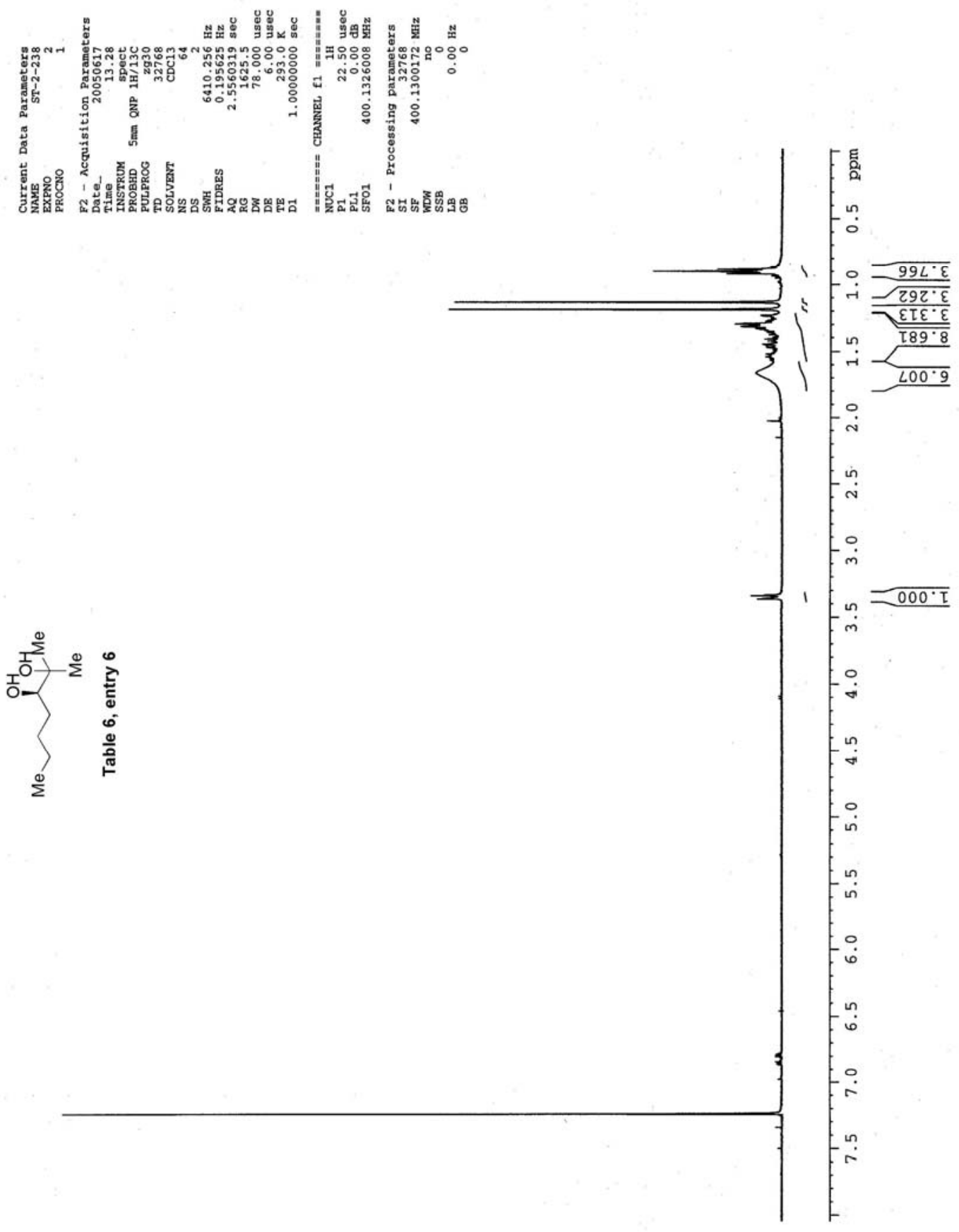

Page S42 


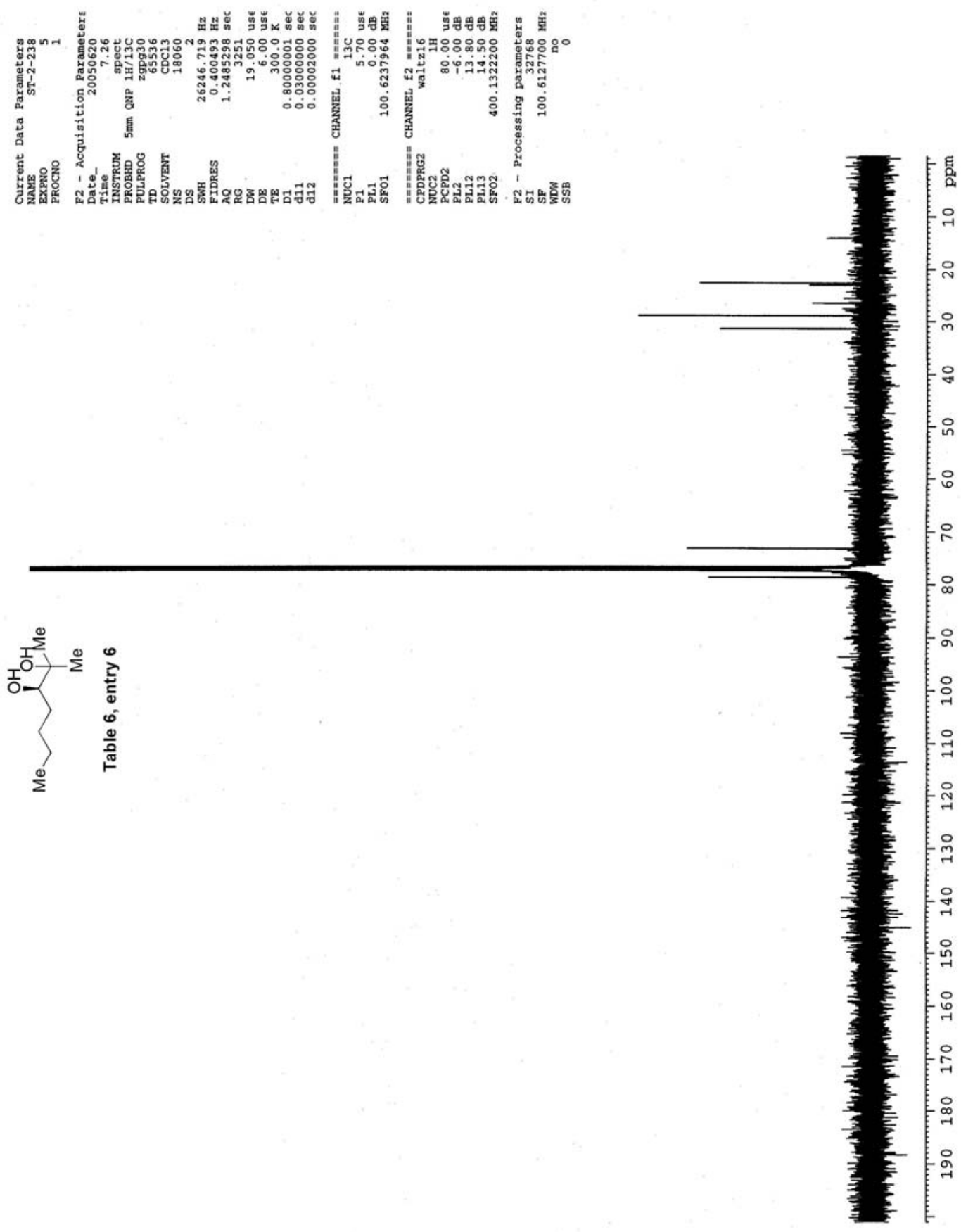

Page S43 


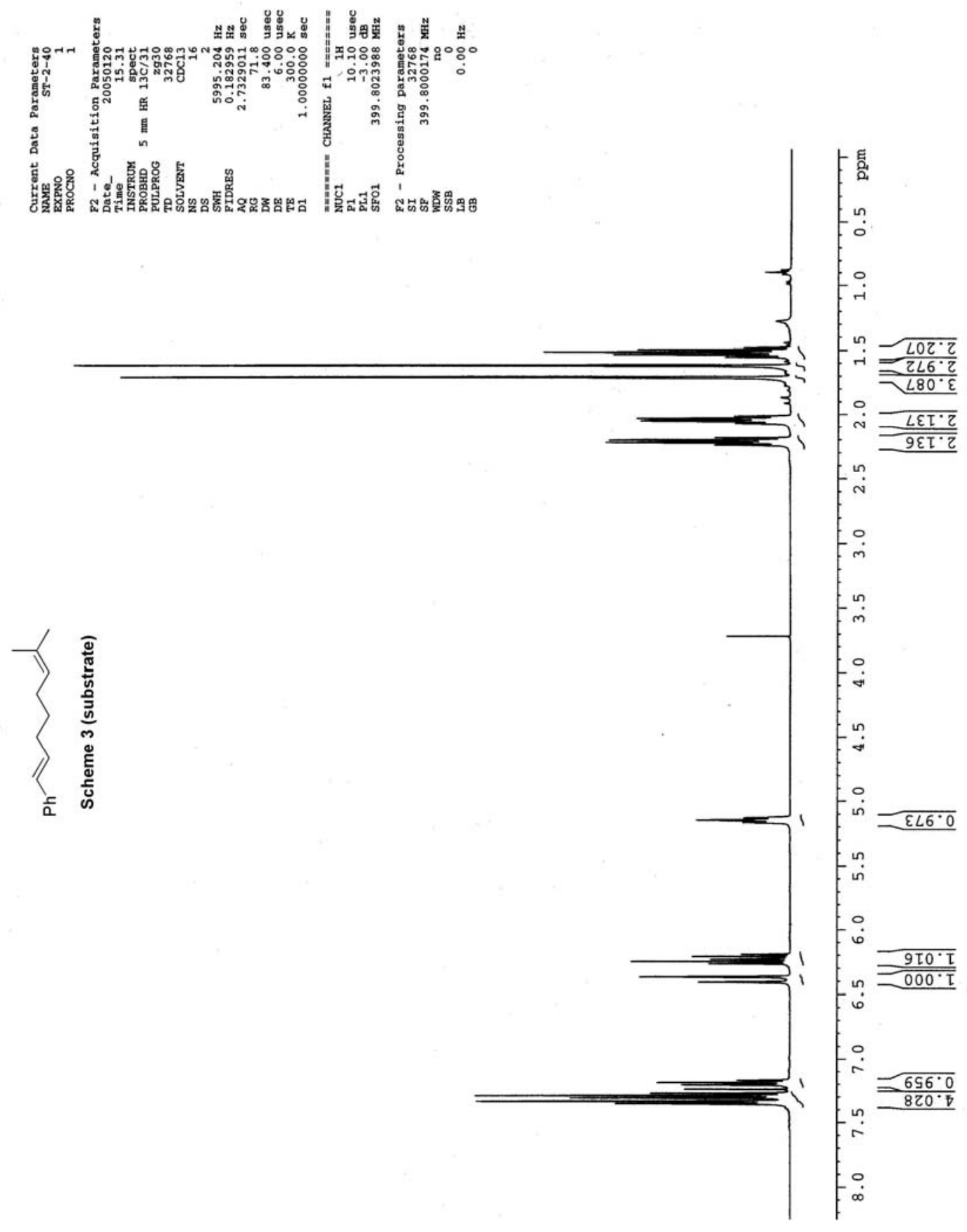




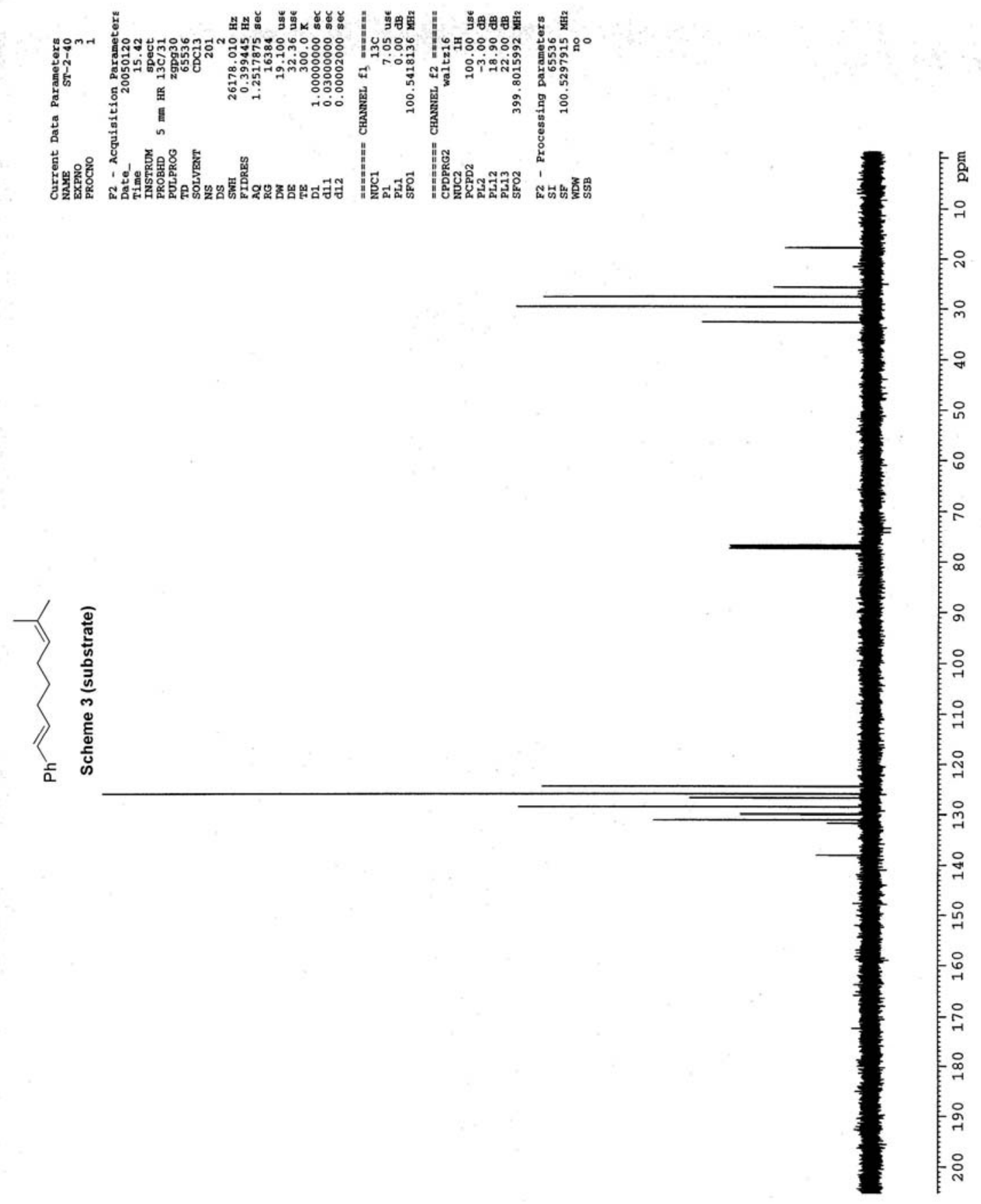

Page S45 


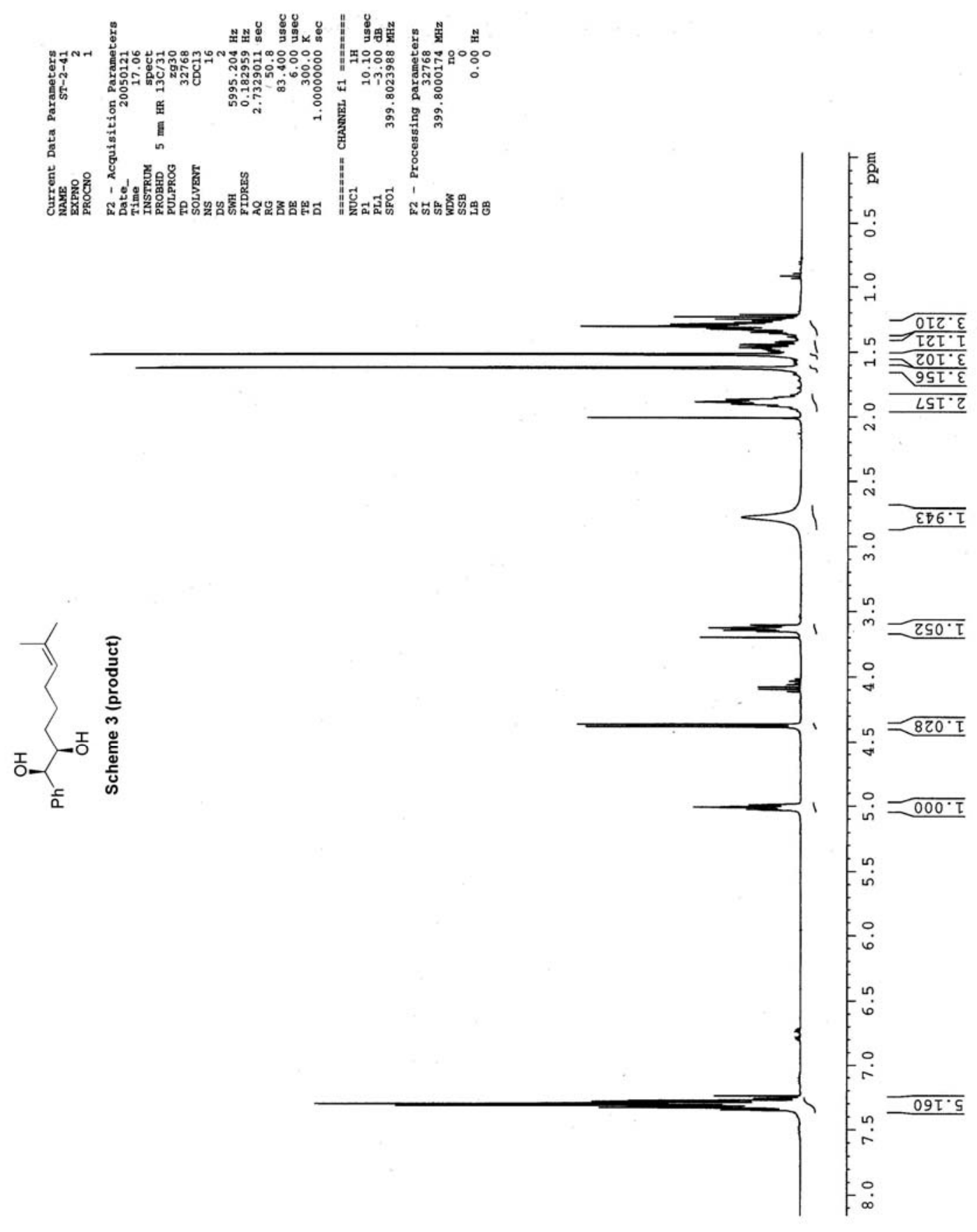




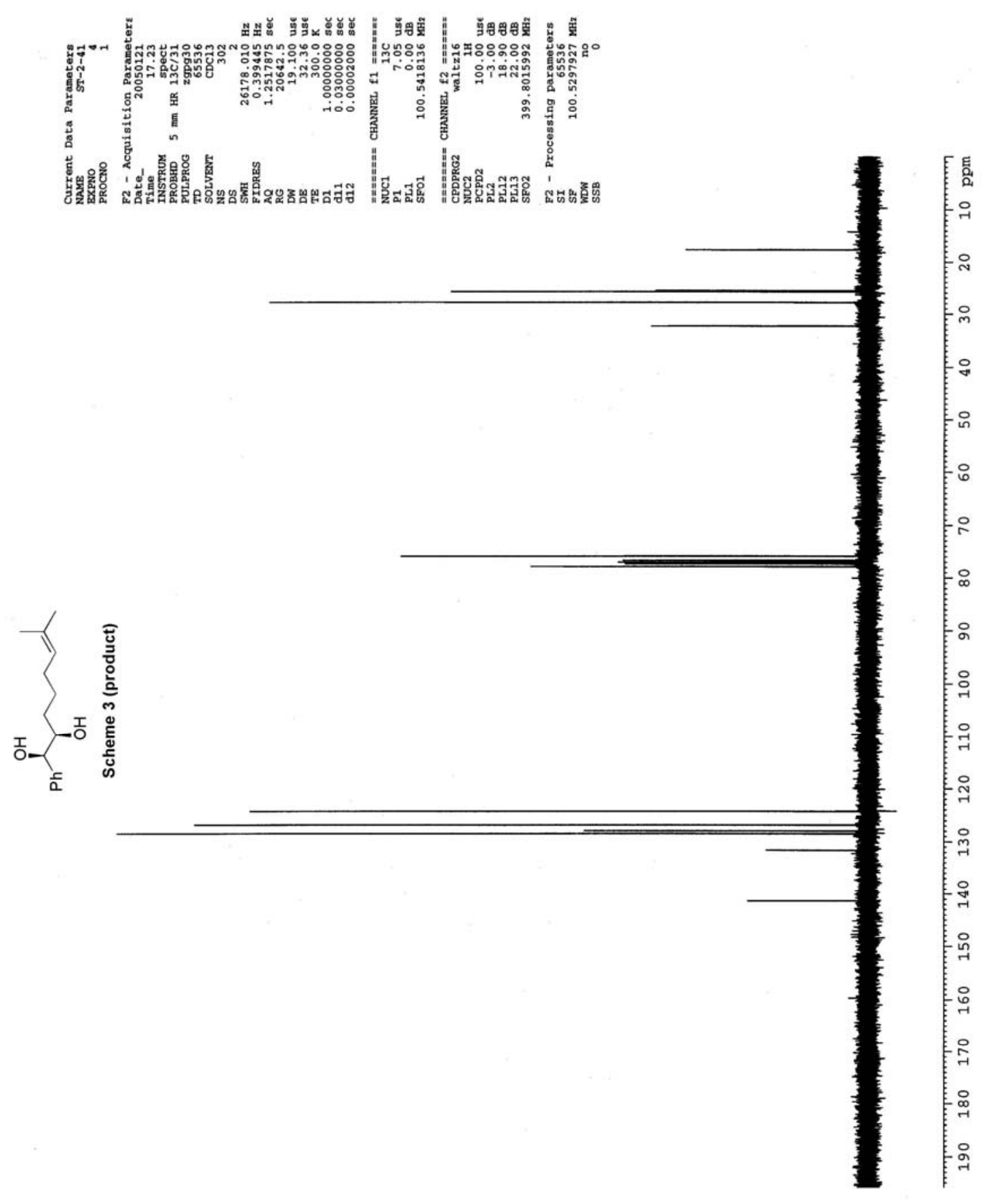

Page S47 
${ }^{1}$ Morgan, J. B.; Miller, S. P.; Morken, J. P. J. Am. Chem. Soc. 2003, 125, 8702.

${ }^{2}$ Miller, S. P.; Morgan, J. B.; Nepveux V, F. J.; Morken, J. P. Org. Lett. 2004, 6, 131.

3 Prepared from trans-6-(tert-butyldiphenylsiloxy)-3-hexene: Han, H.; Cho, C.-W.; Janda, K. D. Chem. Europ. J. 1999, 5, 1565.

${ }^{4}$ Prepared from trans-6-(tert-butyldimethylsiloxy)-3-hexene: Wang, Z.-X.; Tu, Y.; Frohn, M.; Zhang, J.-R.; Shi, Y. J. Am. Chem. Soc. 1997, 119, 11224.

${ }^{5}$ Prepared from trans-1-methoxymethoxy-hex-3-ene: Goff, D. A.; Harris, R. N.; Bottaro, J. C.; Bedford, C. D. J. Org. Chem. 1986, 51, 4711.

${ }^{6}$ Prepared from trans-1-benzyloxy-3-hexene: Azzena, F.; Calvani, F.; Crotti, P.; Gardelli, C.; Macchia, F.; Pineschi, M. Tetrahedron 1995, 51, 10601.

${ }^{7}$ Takada, H.; Nishibayashi, Y.; Uemura, S. J. Chem. Soc. Perkin Trans. 1 1999, 11, 1511.

${ }^{8}$ Prepared from tert-butyl(2,2-dimethylpent-4-enyloxy)diphenylsilane: Chen, G.; Ma, X. S.; Guan, Z. J. Am. Chem. Soc. 2003, 125, 6697.

${ }^{3}$ Prepared from cinnamyl bromide in two steps: Shrestha, K.S.; Honda, K.; Asami, M.; Inoue, S. Bull. Chem. Soc. Jpn. 1999, 72, 73. 